\title{
Thick branes with a nonminimally coupled bulk-scalar field
}

\author{
Heng Guo*, Yu-Xiao Liu ${ }^{\dagger}$, Zhen-Hua Zhao \\ Institute of Theoretical Physics, Lanzhou University, \\ Lanzhou 730000, People's Republic of China
}

\begin{abstract}
In this paper, we investigate thick branes with a nonminimally coupled background scalar field, whose solution is a single-kink or a double-kink. The effects of the nonminimal coupling constant $\xi$ on the structure of the thick branes and the localization of gravity, fermions, scalars and vectors are discussed. It is shown that each brane will split into two sub-branes as increasing the nonminimal coupling constant $\xi$. By investigating the tensor perturbation equations of gravity and the general covariant Dirac equation of fermions, we find that both the gravity zero mode and left-chiral fermion zero mode are localized at the center of the single-kink branes and localized between the two sub-branes generated by the double-kink, which indicates that the constant $\xi$ does not effect the localization of these zero modes. However, the zero mode of scalars is localized on each subbrane (for both single-kink and double-kink branes) when $\xi$ is larger than its critical value $\xi_{0}$. The effects of the nonminimal coupling constant $\xi$ on the resonances of gravity and fermions with finite lifetime on the branes are also discussed.
\end{abstract}

PACS numbers: 04.50.-h, 11.27.+d

\footnotetext{
* guoh2009@lzu.edu.cn

† liuyx@lzu.edu.cn, Corresponding author

$\ddagger$ zhaozhh09@lzu.cn

$\S$ chenfw06@lzu.cn
} 


\section{INTRODUCTION}

The idea that our observed four-dimensional Universe might be a hypersurface, embedded in a higher dimensional space-time (the bulk), provides new insights into solving the gauge hierarchy and cosmological constant problems [1 1 8]. In the Randall-Sundrum (RS) braneworld model, the zero mode of gravity is localized on the brane, which reproduces the standard Newtonian gravity on the brane [5]. But in this model, the brane is very ideal because its thickness is neglected. In the most fundamental theory, there seems to exist a minimum scale of length, thus the thickness of a brane should be considered in more realistic field models. For this reason, more natural thick brane scenarios have been investigated [941]. For some comprehensive reviews about thick branes, see Refs. [42 47].

In brane-world theory, the localization of gravity and various bulk matter fields is a very important issue. In order to recover the effective four-dimensional gravity, the gravity zero mode should be localized on branes. In the other hand, various bulk matter fields should be localized on branes by a natural mechanism, for the purpose to build up the standard model. Generally, the massless scalar fields [48] and graviton [5] could be trapped on branes of different types. Spin-1 Abelian vector fields can be localized on the RS brane in some higher dimensional cases [49] or on the thick de Sitter branes and Weyl thick branes [55]. It is important to study the localization problem of the spin- $1 / 2$ fermions. Without introducing the scalar-fermion coupling, fermions can not be localized on branes in five and six dimensions [48 74]. In some cases, there may exist a single bound state and a continuous gapless spectrum of massive fermion Kaluza-Klein (KK) modes [24, 55]. In some other cases, one can obtain finite discrete KK modes (mass gap) and a continuous gapless spectrum starting at a positive $m^{2}[27,63,64]$.

Generally, including nonlinear terms of the various curvature tensors (Riemann, Ricci, Weyl) and nonminimally coupled terms in the effective action of gravity is a very common trend from quantum field theory and cosmology. These theories cover $f(R)$ modified gravity, the Gauss-Bonnet gravity, scalar-tensor gravity, and so on. On the other hand, in thick brane scenarios, branes are made of background scalar fields, so it is natural to study gravity coupled to background scalars. There are a lot of investigations of thick branes in the case of the minimal coupling, but for the case of the nonminimal coupling, the investigations are limited. Recently, brane-world models with a nonminimally coupled bulk-scalar field, 
via an interaction term $-\frac{1}{2} \xi R \phi^{2}$ with $\phi$ a background scalar field and $\xi$ a nonminimal coupling constant, have been studied. Static solutions of these models have been examined in Refs. [75 78]. The Newton's law for brane models with a nonminimally coupled bulk scalar field was investigated in Ref. [79]. The conditions for localization of gravity for thick brane-worlds with nonminimally coupled term and Gauss-Bonnet term were discussed in Ref. [80]. In Ref. [81], the effective dark energy of the brane-Universe acquires a dynamical nature, as a result of the nonminimal coupling which provides a mechanism for an indirect "bulk-brane interaction" through gravity.

Furthermore, in the brane-world theory, the continuous deformation from a single brane to two sub-branes, by varying parameters, is called the phenomenon of brane splitting [16, 18, 72, 73]. Generally, the single-kink background scalar field can generate the single-brane, and the double-kink scalar field can also result in the double-brane. In this paper, we find that increasing the nonminimal coupling constant $\xi$ can also make the brane splitting from a single-brane to two sub-branes for the single-kink background scalar field. The fermion localization on a split brane has been studied in Refs. [72 74]. Here, for the thick branes generated by a nonminimally coupled background scalar, we find that the different fields are localized on different positions in the extra dimension. This situation is similar to the so-called "split fermion" model [82, 83], which offers the solution to the fast proton decay.

In this paper, we are interested in investigating the structure of thick branes generated by a nonminimally coupled background scalar, and the effects of the nonminimal coupling constant on the localization of gravity and various matter fields. Two general cases of the background scalar field are studied, which are set as a single-kink and a double-kink. We find that the structure of the thick branes is very interesting, and it is related to the nonminimal coupling constant $\xi$. For the single-kink scalar case, as $\xi$ becomes larger, the single brane will split into two sub-branes and the distance of the two sub-branes will increase. For the double-kink scalar case, there are two sub-branes located at both sides of $z=0$, and as $\xi$ becomes larger, the distance of the two sub-branes will also increase. Further, the effects of the nonminimal coupling constant on the localization of gravity and various matter fields are investigated. Comparing to the case of the minimal coupling, the localization of the zero modes of gravity and various matter fields are similar, however, the behavior of the scalar zero mode is different. With the increase of $\xi$, the scalar zero mode is localized first on the center of the two sub-branes for the single-kink case and between them for double- 
kink case, and then on each sub-brane. Furthermore, with the increase of $\xi$, the resonances of gravity will appear, which correspond to gravitons with a finite lifetime on the branes. This phenomenon does not appear in the case of the minimal coupling. The resonances of fermions also exist, and this is similar to the minimal coupling case. The effects of the nonminimal coupling constant $\xi$ on the resonances of gravity and fermions are also discussed.

The organization of this paper is as follows: In Sec. III, the model of thick branes with nonminimally coupled bulk-scalar field in five-dimensional space-time is described, and the structure of the branes is also discussed. Then, in Sec. III, we investigate the localization of gravity on the branes. In Sec.IV], the localization of various bulk matter fields is investigated. Finally, the conclusion is given in Sec. $\mathrm{V}$.

\section{THE STRUCTURE OF THE THICK BRANES WITH A NONMINIMALLY COUPLED BULK-SCALAR FIELD}

We start with the following five-dimensional action of thick branes, which are generated by a real nonminimally coupled scalar field $\phi$,

$$
S=\int d^{5} x \sqrt{-g}\left[F(\phi) R-\frac{1}{2} g^{M N} \partial_{M} \phi \partial_{N} \phi-V(\phi)\right],
$$

where $R$ is the five-dimensional scalar curvature and $F(\phi)$ is chosen as

$$
F(\phi)=\frac{1}{2 \kappa_{5}^{2}}\left(1-\kappa_{5}^{2} \xi \phi^{2}\right),
$$

$\kappa_{5}^{2}=8 \pi G_{5}$ with $G_{5}$ the five-dimensional Newton constant and $\xi$ is a dimensionless coupling constant. The five-dimensional cosmological constant has been included in the scalar potential $V(\phi)$. Here, $F(\phi)$ should be positive, and it is clear that the standard thick brane action is recovered when the coupling constant $\xi=0$.

The Einstein equations corresponding to the action (11) are expressed as follows

$$
R_{M N}-\frac{1}{2} g_{M N} R=T_{M N}
$$

with $T_{M N}$ the energy-momentum tensor for the scalar field:

$$
\begin{aligned}
T_{M N}= & \partial_{M} \phi \partial_{N} \phi-g_{M N}\left[\frac{1}{2} g^{P Q} \partial_{P} \phi \partial_{Q} \phi+V(\phi)\right] \\
& +2 \nabla_{M} \nabla_{N} F(\phi)-2 g_{M N} \square F(\phi) \\
& +(1-2 F(\phi))\left(R_{M N}-\frac{1}{2} g_{M N} R\right),
\end{aligned}
$$


where $\square$ is the five-dimensional d'Alembertian operator. We have set $\kappa^{2}=1$.

The equation of motion for the scalar field reads

$$
\square \phi+\frac{d F(\phi)}{d \phi} R-\frac{d V(\phi)}{d \phi}=0 .
$$

The above equation and the Einstein equations (3) are not independent [75].

The line-element for the background space-time describing a thick flat brane is assumed as

$$
d s^{2}=g_{M N} d x^{M} d x^{N}=\mathrm{e}^{2 A(y)} \eta_{\mu \nu} d x^{\mu} d x^{\nu}+d y^{2}
$$

where $\mathrm{e}^{2 A(y)}$ is the warp factor, $\eta_{\mu \nu}=\operatorname{diag}(-1,+1,+1,+1)$ is the Minkowski metric, and $y$ stands for the extra coordinate. We suppose that the scalar field is considered to be a function of $y$ only, i.e., $\phi=\phi(y)$. From Eqs (3)-(6)

$$
\begin{aligned}
3\left(1-\xi \phi^{2}\right)\left(A^{\prime \prime}+2 A^{2}\right)+ & \left(\frac{1}{2}-2 \xi\right) \phi^{\prime 2}+V(\phi)-2 \xi \phi \phi^{\prime \prime}-6 \xi A^{\prime} \phi \phi^{\prime}=0, \\
6\left(1-\xi \phi^{2}\right) A^{\prime 2}-\frac{1}{2} \phi^{2}+V(\phi)-8 \xi A^{\prime} \phi \phi^{\prime} & =0, \\
-\phi^{\prime \prime}-4 A^{\prime} \phi^{\prime}-\xi\left(8 A^{\prime \prime}+20 A^{\prime 2}\right) \phi+\frac{d V(\phi)}{d \phi} & =0,
\end{aligned}
$$

where the prime stands for the derivative with respect to the extra coordinate. A static analytical solution, for a narrow range of the coupling constant values $0<\xi<\frac{1}{6}$, has been studied in Ref. [77]. In this paper, we will investigate the effect of the coupling constant $\xi$ on the brane structure and the localization of gravity and various spin fields in some general situations.

For the sake of convenience of obtaining the mass-independent localization potential for gravitons, we will follow Ref. [5] and change the metric given in (66) to the conformally flat form

$$
d s^{2}=\mathrm{e}^{2 A(z)}\left(\eta_{\mu \nu} d x^{\mu} d x^{\nu}+d z^{2}\right)
$$

by performing the coordinate transformation

$$
d z=\mathrm{e}^{-A(y)} d y
$$

The equations of the motion for the background scalar field $\phi(z)$ and the warp factor $A(z)$ 
in the $z$ coordinate can be written as

$$
\begin{aligned}
& 3\left(1-\xi \phi^{2}\right)\left(A^{\prime 2}+A^{\prime \prime}\right)+\frac{1}{2} \phi^{2}+\mathrm{e}^{2 A} V(\phi)-2 \xi \phi^{\prime 2}-2 \xi \phi \phi^{\prime \prime}-4 \xi \phi \phi^{\prime \prime}=0, \\
& 6\left(1-\xi \phi^{2}\right) A^{\prime 2}-\frac{1}{2} \phi^{\prime 2}+\mathrm{e}^{2 A} V(\phi)-8 \xi \phi A^{\prime} \phi^{\prime}=0, \\
& \phi^{\prime \prime}+3 A^{\prime} \phi^{\prime}+4 \xi \phi\left(3 A^{2}+2 A^{\prime \prime}\right)-\mathrm{e}^{2 A} \frac{d V(\phi)}{d \phi}=0 .
\end{aligned}
$$

As we have already mentioned, the above equations are not independent. Hence, we choose to solve Eq. (10b) and the following equation, which can be obtained from (10a) and (10b),

$$
\begin{gathered}
3\left(1-\xi \phi^{2}\right)\left(A^{\prime \prime}-A^{2}\right)+4 \xi \phi A^{\prime} \phi^{\prime} \\
+(1-2 \xi) \phi^{\prime 2}-2 \xi \phi \phi^{\prime \prime}=0 .
\end{gathered}
$$

By considering that the energy density $T_{00}(z)$ should vanish as $z \rightarrow \pm \infty$, the background scalar field should satisfy $\phi(z \rightarrow \pm \infty)<\infty$ and $\phi^{\prime}(z \rightarrow \pm \infty)<\infty$, so the background scalar field is naturally considered as a kink solution

$$
\phi(z)=\phi_{0} \tanh ^{k}(b z)
$$

where $\phi_{0}$ and $b$ are positive real parameters, and the parameter $k$ is an positive odd number. Here we just only consider two cases: the $k=1$ single-kink solution and the $k=3$ doublekink solution. The background scalar has the following behavior at $z=0$ and $z \rightarrow \pm \infty$ :

$$
\phi(0)=0, \quad \phi(z \rightarrow \pm \infty)= \pm \phi_{0}
$$

Because $F(\phi)>0$ and $\phi \leq \phi_{0}$, the coupling constant $\xi<\frac{1}{\phi_{0}}$. It is very difficult to solve Eq. (11) by analytical method, so we will numerically solve it. First, we will analyze the asymptotic behavior of the warp factor at $z \rightarrow \pm \infty$. When $z \rightarrow \pm \infty$, we have for the scalar field that $\phi \rightarrow \pm \phi_{0}, \phi^{\prime} \rightarrow 0$ and $\phi^{\prime \prime} \rightarrow 0$. Hence, Eq. (11) reduces to $A^{\prime \prime}(z)-$ $A^{\prime 2}(z)=0$ as $z \rightarrow \pm \infty$, from which the asymptotical form of the warp factor can be given as $\mathrm{e}^{A(z)}=\frac{1}{c+b|z|} \simeq \frac{1}{b|z|}$, which is the same as the RS brane-world. Next, we want to obtain the approximate solution of Eq. (11) in the vicinity of $z=0$. We expand the background scalar field $\phi(z)$ at $z=0$ :

$$
\phi(z)=\phi_{0}(b z)^{k}-\frac{1}{3} \phi_{0} k(b z)^{k+2}+\mathcal{O}\left((b z)^{k+4}\right) .
$$


For $k=1$, by using $\phi(z)=\phi_{0}(b z)-\frac{1}{3} \phi_{0}(b z)^{3}+\mathcal{O}\left((b z)^{5}\right)$, the behavior of the warp factor in the vicinity of $z=0$, from Eq. (11), can be expressed as

$$
A(z)=\frac{1}{6} \phi_{0}^{2}(2 \xi-1)(b z)^{2}+\mathcal{O}\left((b z)^{3}\right) .
$$

We find that the behavior of the warp factor in the vicinity of $z=0$ is related closely to a critical coupling constant $\xi_{0}=\frac{1}{2}$. When small $\xi$ with $\xi<\xi_{0}$, the maximum of the warp factor $\mathrm{e}^{2 A(z)}$ is at $z=0$, and this will correspond to one brane located at $z=0$. When $\xi$ is closer to $\xi_{0}$, we will see that the thick brane will split into two sub-branes. When $\xi>\xi_{0}$, the maxima of the warp factor are at both sides of $z=0$ and this will result in the increase of the distance of the two sub-branes.

For $k=3$, the scalar $\phi(z)$ can be expressed as $\phi(z)=\phi_{0}(b z)^{3}+\mathcal{O}\left((b z)^{5}\right)$ at $z=0$. And the warp factor can be described as

$$
A(z)=\frac{1}{30} \phi_{0}^{2}(10 \xi-3)(b z)^{6}+\mathcal{O}\left((b z)^{7}\right) .
$$

Here the critical coupling constant is $\xi_{0}=\frac{3}{10}$. The behavior of the warp factor is the same as the case $k=1$. However, there are always two sub-branes for any $\xi$ because the scalar is a double-kink.

Eq. (11) can be solved numerically with the following initial conditions:

$$
A(0)=A^{\prime}(0)=0
$$

The shapes of the warp factor $\mathrm{e}^{2 A}$ and the energy density $T_{00}(z)$ are shown in Fig. 1 and Fig. 2 for $k=1$ and $k=3$, respectively. For $k=1$, when $\xi$ is very small, there is only one brane located at $z=0$, and when increasing $\xi$, the brane gradually splits two sub-branes located at both sides of $z=0$. The distance of the two sub-branes increases with $\xi$. For $k=3$, there are two sub-branes located at both sides of $z=0$ even for small $\xi$, and the distance of the two sub-branes also increases with $\xi$.

The scalar potential $V(\phi)$ can also be solved numerically by Eqs. (10b) and (11), and it is shown in Fig. 1(d) and Fig. 2(d) for single-kink $(k=1)$ and double-kink $(k=3)$, respectively. Because the range of the background scalar $\phi(z)$ is from $-\phi_{0}$ to $+\phi_{0}$, the scalar potential $V(\phi)$ only can be given between $V\left(-\phi_{0}\right)$ and $V\left(+\phi_{0}\right)$ by numerical method. It is shown that the scalar potential is a double well potential. When the coupling constant $\xi=0$, the vacua of the potential are at $\pm \phi_{0}$, and the background scalar (single- or doublekink) connects two vacua of the double well potential. When $\xi>0$, the two vacua are not 
at $\pm \phi_{0}$, and this can also be obtained by analyzing the field equations. From Eq. (10c), the following expression is obtained easily

$$
\frac{d V(\phi)}{\phi}=\mathrm{e}^{-2 A}\left[\phi^{\prime \prime}+3 A^{\prime} \phi^{\prime}+4 \xi \phi\left(3 A^{\prime 2}+2 A^{\prime \prime}\right)\right] .
$$

Considering that the background scalar field and the warp factor have the following asymptotic behavior $\phi( \pm \infty) \rightarrow \pm \phi_{0}, \phi^{\prime}( \pm \infty) \rightarrow \pm 4 k \phi_{0} \mathrm{e}^{-2 z}, \phi^{\prime \prime}( \pm \infty) \rightarrow \mp 8 k \phi_{0} \mathrm{e}^{-2 z}$, $A( \pm \infty) \rightarrow-\ln b|z|, A^{\prime}( \pm \infty) \rightarrow-\frac{1}{|z|}$ and $A^{\prime \prime}( \pm \infty) \rightarrow \frac{1}{z^{2}}$, the above expression can be reduced at infinity to

$$
\frac{d V(\phi)}{d \phi} \rightarrow \pm 20 \xi b^{2} \phi_{0}
$$

and it is clear that the two vacua of the potential $V(\phi)$ are at $\pm \phi_{0}$ only for $\xi=0$. At $\phi=0$, the scalar potential $V(\phi)$ has different behaviors for $k=1$ and $k=3$. For $k=1$, when the coupling constant $\xi$ is small, the scalar potential $V(\phi)$ has a local maximum at $\phi=0$, which is similar to the $\phi^{4}$ model in particle physics, and when $\xi$ becomes large and closes to $\frac{1}{\phi_{0}}=1$, the potential at $\phi=0$ turns to a local minimum, which is similar to $\phi^{6}$ model in particle physics. For $k=3, V(\phi)$ always has a local minimum at $\phi=0$, which is the same as $\phi^{6}$ model.

\section{LOCALIZATION OF GRAVITY ON THE THICK BRANES}

In this section we will investigate the localization of the gravity on the branes, by the linearized equations for the metric fluctuations. From the Einstein equations (3), we can obtain the alternative form of the Einstein equations:

$$
2 F(\phi) R_{M N}=\widetilde{T}_{M N}-\frac{1}{3} g_{M N} \widetilde{T}
$$

where $\widetilde{T}=g^{M N} \widetilde{T}_{M N}$ and

$$
\begin{gathered}
\widetilde{T}_{M N}=\partial_{M} \phi \partial_{N} \phi-g_{M N}\left[\frac{1}{2} g^{P Q} \partial_{P} \phi \partial_{Q} \phi+V(\phi)\right] \\
+2 \nabla_{M} \nabla_{N} F(\phi)-2 g_{M N} \square F(\phi) .
\end{gathered}
$$

From the above equations, we will obtain the linearized equations for the metric fluctuations $h_{M N}$.

Under the axial gauge conditions $h_{5 M}=0$, the total metric can be written in the form

$$
d s^{2}=\mathrm{e}^{2 A(y)}\left(\eta_{\mu \nu}+h_{\mu \nu}\right) d x^{\mu} d x^{\nu}+d y^{2} .
$$




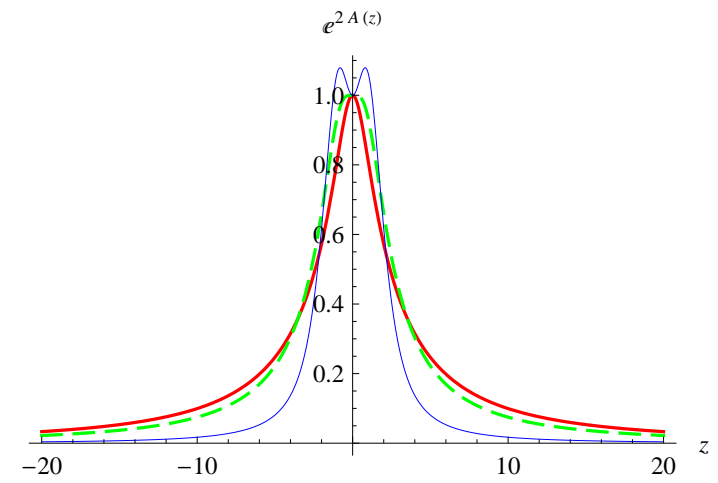

(a) $k=1$

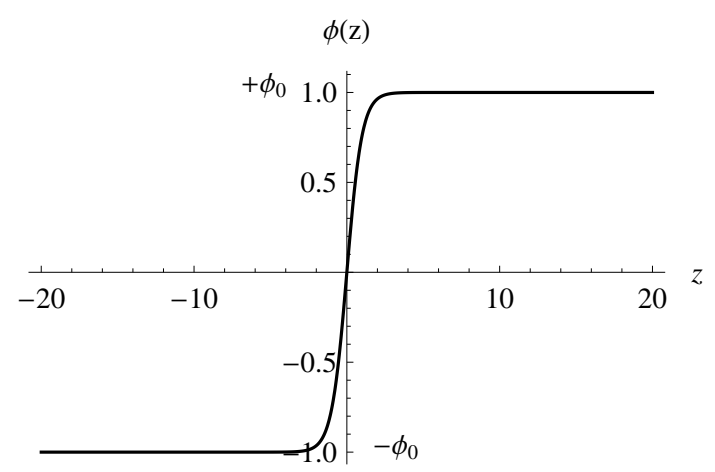

(c) $k=1$

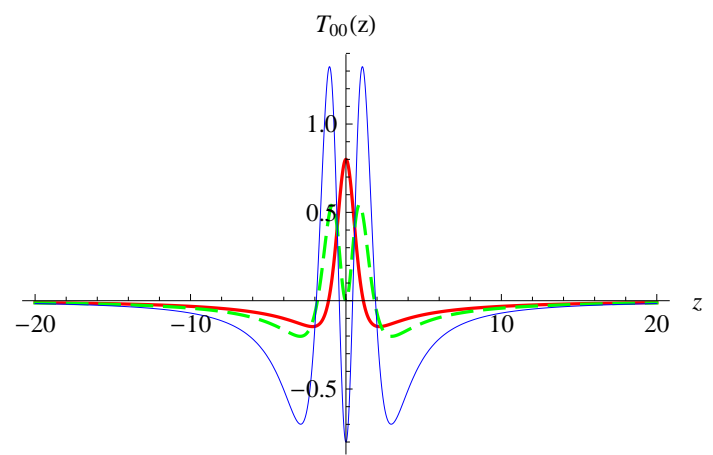

(b) $k=1$

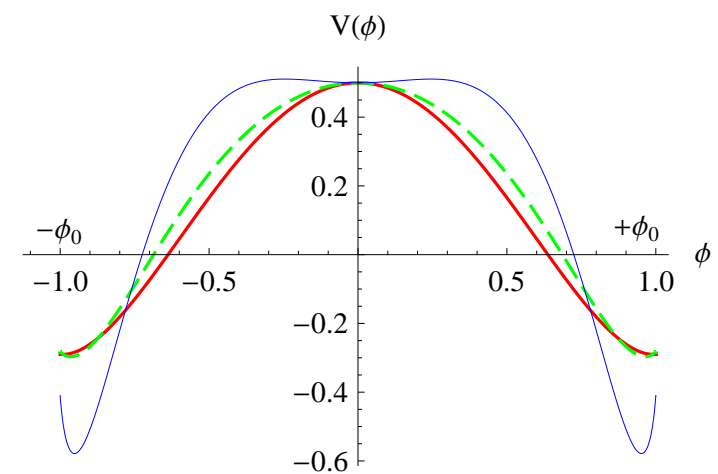

(d) $k=1$

FIG. 1: The shapes of the warp factor $\mathrm{e}^{2 A}$, the energy density $T_{00}(z)$, the background scalar field $\phi(z)$ and scalar potential $V(\phi)$. The parameters are set to $\xi=0.1$ for the thick red line, $\xi=0.5$ for the dashed green line, and $\xi=0.9$ for the thin blue line. The other parameters are set as $\phi_{0}=1$, $b=1$, and $k=1$.

The Ricci tensor can be computed from the metric (22)

$$
R_{M N}=R_{M N}^{(0)}+R_{M N}^{(1)}+\cdots
$$

where the zero order terms are

$$
\begin{aligned}
& R_{\mu \nu}^{(0)}=-\mathrm{e}^{2 A}\left(A^{\prime \prime}+4 A^{2}\right) \eta_{\mu \nu}, \quad R_{55}^{(0)}=-4\left(A^{\prime \prime}+A^{\prime 2}\right), \\
& R_{5 \mu}^{(0)}=0
\end{aligned}
$$




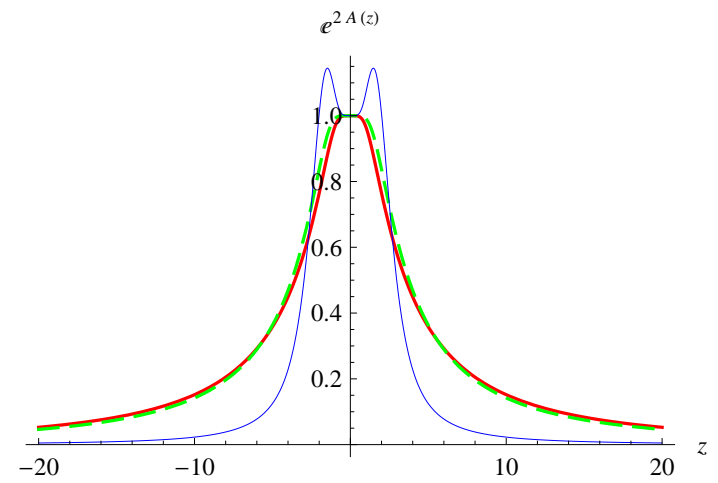

(a) $k=3$

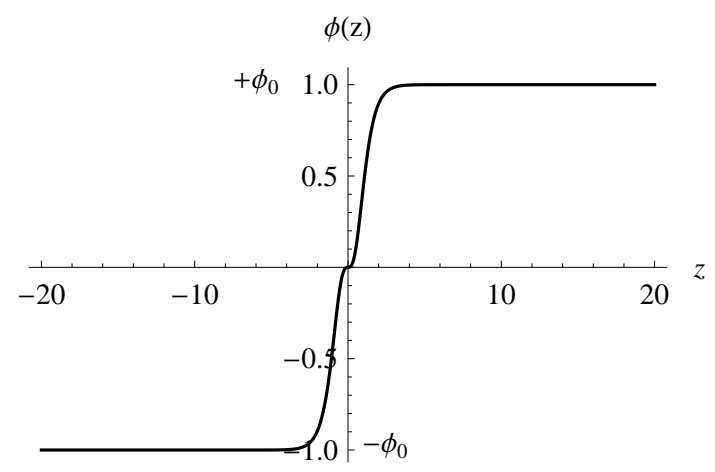

(c) $k=3$

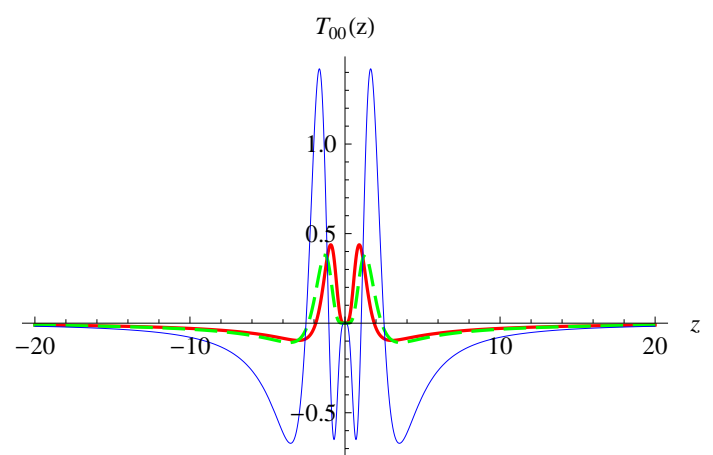

(b) $k=3$

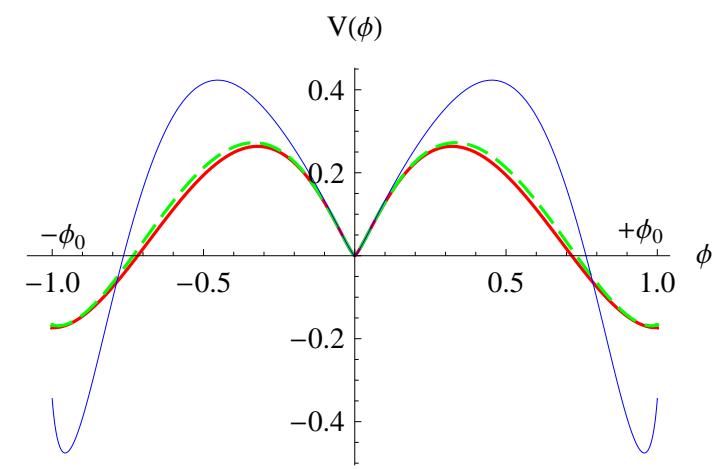

(d) $k=3$

FIG. 2: The shapes of the warp factor $\mathrm{e}^{2 A}$, the energy density $T_{00}(z)$, the background scalar field $\phi(z)$ and scalar potential $V(\phi)$. The parameters are set to $\xi=0.1$ for the thick red line, $\xi=0.3$ for the dashed green line, and $\xi=0.9$ for the thin blue line. The other parameters are set as $\phi_{0}=1$, $b=1$, and $k=3$.

and the first order terms are

$$
\begin{aligned}
R_{\mu \nu}^{(1)}= & -\mathrm{e}^{2 A}\left(\frac{1}{2} \partial_{y}^{2}+2 A^{\prime} \partial_{y}+A^{\prime \prime}+4 A^{\prime 2}\right) h_{\mu \nu} \\
& -\frac{1}{2} \square^{(4)} h_{\mu \nu}-\frac{1}{2} \eta_{\mu \nu} \mathrm{e}^{2 A} A^{\prime} \partial_{y}\left(\eta^{\alpha \beta} h_{\alpha \beta}\right) \\
& -\frac{1}{2} \eta^{\alpha \beta}\left(\partial_{\mu} \partial_{\nu} h_{\alpha \beta}-\partial_{\mu} \partial_{\alpha} h_{\nu \beta}-\partial_{\nu} \partial_{\alpha} h_{\mu \beta}\right), \\
R_{55}^{(1)}= & -\frac{1}{2}\left(\partial_{y}^{2}+2 A^{\prime} \partial_{y}\right) \eta^{\mu \nu} h_{\mu \nu}, \\
R_{5 \mu}^{(1)}= & \frac{1}{2} \eta^{\alpha \beta} \partial_{y}\left(\partial_{\alpha} h_{\mu \beta}-\partial_{\mu} h_{\alpha \beta}\right) .
\end{aligned}
$$


And we can also obtain

$$
\widetilde{T}_{M N}=\widetilde{T}_{M N}^{(0)}+\widetilde{T}_{M N}^{(1)}+\cdots
$$

with

$$
\begin{aligned}
\widetilde{T}_{\mu \nu}^{(0)}=-\mathrm{e}^{2 A} \eta_{\mu \nu}[6 & \frac{d F}{d \phi} \phi^{\prime} A^{\prime}+2 \frac{d^{2} F}{d \phi^{2}} \phi^{\prime 2} \\
& \left.+2 \frac{d F}{d \phi} \phi^{\prime \prime}+\frac{1}{2} \phi^{\prime 2}+V(\phi)\right], \\
\widetilde{T}_{55}^{(0)}= & \frac{1}{2} \phi^{\prime 2}-V(\phi)-8 \frac{d F}{d \phi} \phi^{\prime} A^{\prime}, \quad \widetilde{T}_{5 \mu}^{(0)}=0,
\end{aligned}
$$

and

$$
\begin{aligned}
\widetilde{T}_{\mu \nu}^{(1)}= & -\mathrm{e}^{2 A} h_{\mu \nu}\left[6 \frac{d F}{d \phi} \phi^{\prime} A^{\prime}+2 \frac{d^{2} F}{d \phi^{2}} \phi^{2}+2 \frac{d F}{d \phi} \phi^{\prime \prime}+\frac{1}{2} \phi^{\prime 2}\right. \\
& +V(\phi)]+\mathrm{e}^{2 A} \frac{d F}{d \phi} \phi^{\prime} h_{\mu \nu}^{\prime}-\mathrm{e}^{2 A} \eta_{\mu \nu} \frac{d F}{d \phi} \phi^{\prime} h^{\prime}, \\
\widetilde{T}_{55}^{(1)}= & -\frac{d F}{d \phi} \phi^{\prime} h^{\prime}, \quad \widetilde{T}_{5 \mu}^{(1)}=0,
\end{aligned}
$$

where $h=\eta^{\mu \nu} h_{\mu \nu}$. From above equations and considering the fluctuations $h_{\mu \nu}$ satisfy the transverse $\partial^{\mu} h_{\mu \nu}=0$ and traceless $h=\eta^{\mu \nu} h_{\mu \nu}=0$ conditions, we can obtain the following equations

$$
\left[\partial_{y}^{2}+Q^{\prime}(y) \partial_{y}+\mathrm{e}^{-2 A} \square^{(4)}\right] h_{\mu \nu}^{T T}(x, y)=0
$$

where we have set

$$
Q(y)=4 A(y)+\ln F(\phi(y))
$$

Hence, as we required in Sec. II. $F(\phi(y))$ should satisfy $F(\phi(y))>0$. By using the decomposition $h_{\mu \nu}^{T T}(x, y)=\mathrm{e}^{i p x} \tilde{h}_{\mu \nu}(y)$, the above equation can be reexpressed as

$$
\left[\partial_{y}^{2}+Q^{\prime}(y) \partial_{y}+m^{2} \mathrm{e}^{-2 A}\right] \tilde{h}_{\mu \nu}(y)=0
$$

with $p^{\mu} p_{\mu}=-m^{2}$ the four-dimensional mass of the gravitons.

By using the conformally flat metric (8), Eq. (32) can be rewritten as

$$
\left[\partial_{z}^{2}+\widetilde{Q}^{\prime}(z) \partial_{z}+m^{2}\right] \tilde{h}_{\mu \nu}(z)=0
$$


where $\widetilde{Q}(z)=3 A(z)+\ln F(\phi(z))$. By using the transformation $\tilde{h}_{\mu \nu}(z)=\mathrm{e}^{-\widetilde{Q} / 2} \varphi_{\mu \nu}(z)$, Eq. (35) can be expressed as the Schrödinger equation

$$
\left[-\partial_{z}^{2}+V_{\mathrm{QM}}(z)\right] \varphi(z)=m^{2} \varphi(z)
$$

where $\varphi_{\mu \nu}(z)$ has been replaced by $\varphi(z)$ for simplicity and the localization potential is read as

$$
V_{\mathrm{QM}}(z)=\frac{1}{2} \widetilde{Q}^{\prime \prime}(z)+\frac{1}{4} \widetilde{Q}^{\prime 2}(z)
$$

where the prime denotes the derivative with respect to z. Eq. (36) can be rewritten alternatively as

$$
\left[\partial_{z}+\frac{1}{2} \widetilde{Q}^{\prime}(z)\right]\left[\partial_{z}-\frac{1}{2} \widetilde{Q}^{\prime}(z)\right] \varphi(z)=-m^{2} \varphi(z)
$$

so it will have a complete system of eigenstates with nonnegative eigenvalues, i.e., $m^{2} \geq 0$.

By setting $m=0$, the massless wave function (the zero mode) can be obtained

$$
\varphi_{0}(z) \propto \mathrm{e}^{\frac{3}{2} A(z)} F^{\frac{1}{2}}(\phi(z))
$$

If the zero mode satisfies the normalization condition

$$
\int_{-\infty}^{+\infty} \varphi_{0}^{2}(z) d z=\int_{-\infty}^{+\infty} \mathrm{e}^{3 A(z)} F(\phi(z)) d z<\infty,
$$

the zero mode is localized on the brane.

From the solutions which we have shown in Sec. II, the potential of gravity KK modes $V_{\mathrm{QM}}$ (37) and the gravity zero mode (39) are also can be obtained, which are shown in Figs. 3 and 4.

By considering the asymptotic behavior of the warp factor and the background scalar, the asymptotic behavior of the potential as $z \rightarrow \pm \infty$ is

$$
V_{\mathrm{QM}}(z \rightarrow \pm \infty) \rightarrow 0
$$

which is similar to a volcano-type potential. The asymptotic behavior of the gravity zero mode is

$$
\varphi_{0}(z \rightarrow \pm \infty) \propto \frac{1}{2}\left(1-\xi \phi_{0}^{2}\right)^{\frac{1}{2}}\left(\frac{1}{b|z|}\right)^{\frac{3}{2}} .
$$


and the normalization condition (40) is equivalent to the following condition

$$
\int_{1}^{+\infty} \frac{1}{4}\left(1-\xi \phi_{0}^{2}\right)\left(\frac{1}{b|z|}\right)^{3} d z=\frac{1-\xi \phi_{0}^{2}}{8 b^{3}}<\infty
$$

so it is clear that the normalization condition is satisfied. For $k=1$, when $\xi$ is smaller than the critical coupling constant $\xi_{0}$, the zero mode is localized on the single brane, and when $\xi$ is close to $\xi_{0}$ or $\xi>\xi_{0}$, the zero mode is localized at the center of the two sub-branes. For $k=3$, the zero mode is localized between the two sub-branes. The massive gravity KK modes are the continuous spectrum wave functions with $m^{2}>0$, and asymptotically turn into plane waves.

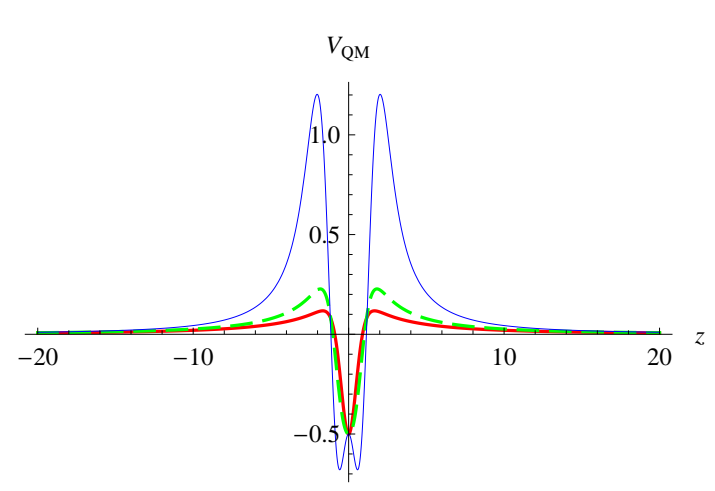

(a) $k=1$

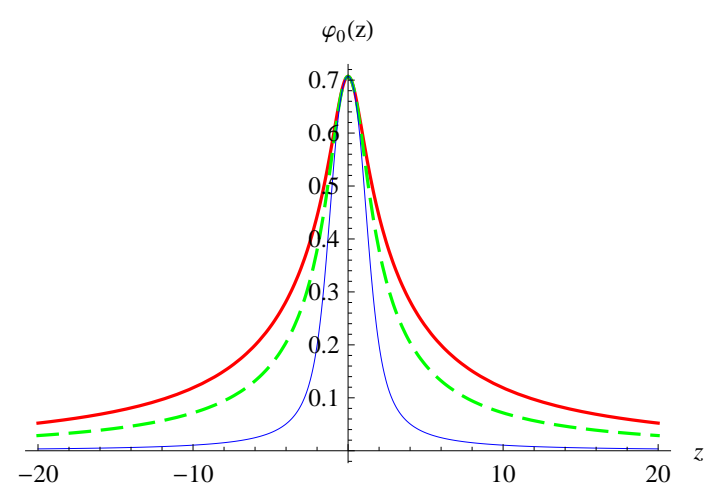

(b) $k=1$

FIG. 3: The shapes of the potential of gravity KK modes $V_{\mathrm{QM}}(z)$ and the gravity zero mode $\varphi_{0}(z)$. The parameters are set to $\xi=0.1$ for the thick red line, $\xi=0.5$ for the dashed green line, and $\xi=0.9$ for the thin blue line. The other parameters are set as $\phi_{0}=1, b=1$, and $k=1$.

Generally, the volcano-type potential implies that there may exist resonant states, which are quasilocalized on the branes. So we will study whether the resonant states exist in this system. From Figs. 3 and 4 , it is seen that with the coupling constant $\xi$ becoming larger, the height of the potential $V_{\mathrm{QM}}$ gets higher, with which the resonant states could be obtained more easily. When the coupling constant $\xi=0.9$ and other parameters are set as $\phi_{0}=1$ and $b=1$, there is only one resonant state for the double-kink background scalar field. However, we find that when the coupling constant $\xi=0.99$ and other parameters are set as $\phi_{0}=1$ and $b=1$, there is a resonant KK mode for the single-kink background scalar field, and two resonant KK modes for the double-kink background scalar field. Because resonant states are oscillating when far away from the brane along the extra dimension, they can not be 


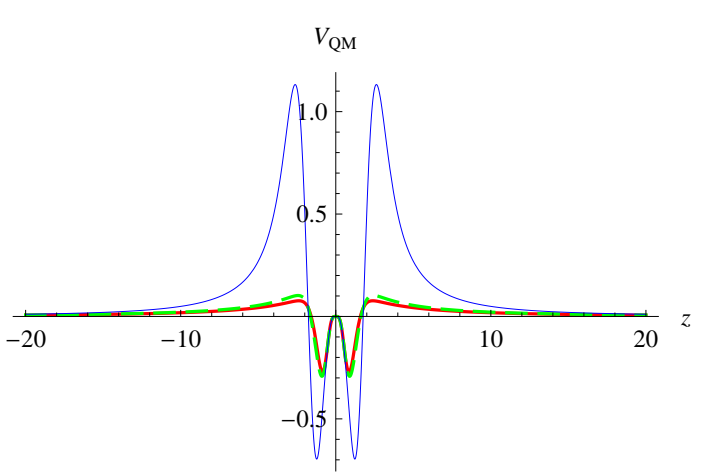

(a) $k=3$

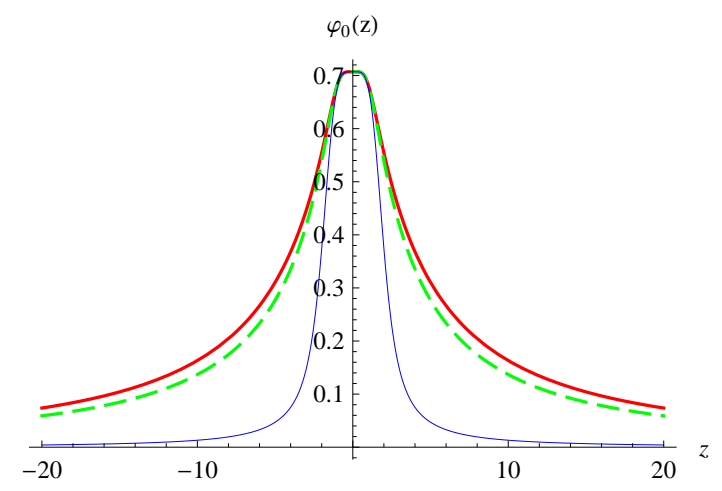

(b) $k=3$

FIG. 4: The shapes of the potential of gravity KK modes $V_{\mathrm{QM}}(z)$ and the gravity zero mode $\varphi_{0}(z)$. The parameters are set to $\xi=0.1$ for the thick red line, $\xi=0.3$ for the dashed green line, and $\xi=0.9$ for the thin blue line. The other parameters are set as $\phi_{0}=1, b=1$, and $k=3$.

normalized. As Ref. [60], we use the function

$$
P_{\mathrm{G}}\left(m^{2}\right)=\frac{\int_{-z_{b}}^{z_{b}}|\varphi(z)|^{2} d z}{\int_{-z_{\max }}^{z_{\max }}|\varphi(z)|^{2} d z}
$$

to describe the relative probability for finding the resonances on a thick brane. Here $2 z_{b}$ is about the width of the thick brane and $z_{\max }$ is set to $z_{\max }=10 z_{b}$. It is clear that for KK modes with $m^{2} \gg V_{\mathrm{QM}}^{\max }\left(V_{\mathrm{QM}}^{\max }\right.$ is the maximum value of $\left.V_{\mathrm{QM}}\right)$, which are approximately taken as plane waves, the value of $P_{\mathrm{G}}\left(\mathrm{m}^{2}\right)$ will trend to $\frac{1}{10}$.

The profiles of $P_{\mathrm{G}}$ corresponding to the coupling constant $\xi=0.9$ for single-kink scalar field $(k=1)$ and double-kink background scalar field $(k=3)$ are shown in Fig. 5 for the thick brane, and $P_{\mathrm{G}}$ corresponding to $\xi=0.99$ for $k=1$ and $k=3$ are shown in Fig. 6. In these figures, each peak corresponds to a resonant state. When $\xi=0.9$, it is seen that only for the double-kink background scalar field there is a resonant KK mode. When $\xi=0.99$, there are one and two resonant KK modes for single-kink and double-kink background scalars, respectively. Hence, we come to the conclusion that if the coupling constant $\xi$ becomes larger, the number of the resonant gravity KK modes will increase. As Ref. [15], we estimate the lifetime $\tau$ of a resonant state as $\tau \sim \Gamma^{-1}$ with $\Gamma=\delta m$ being the full width at half maximum of the peak. Then the mass $m$, width $\Gamma$ and lifetime $\tau$ of the resonant KK modes are listed in Table I, and the resonant KK modes are shown in Figs. 7, 8 and 9 


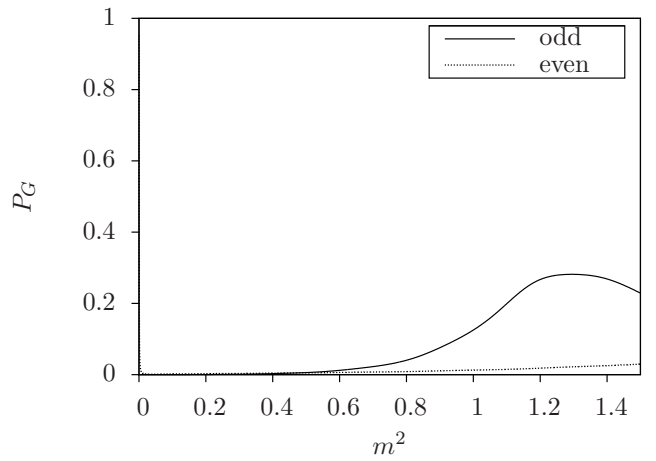

(a) $\xi=0.9, k=1$

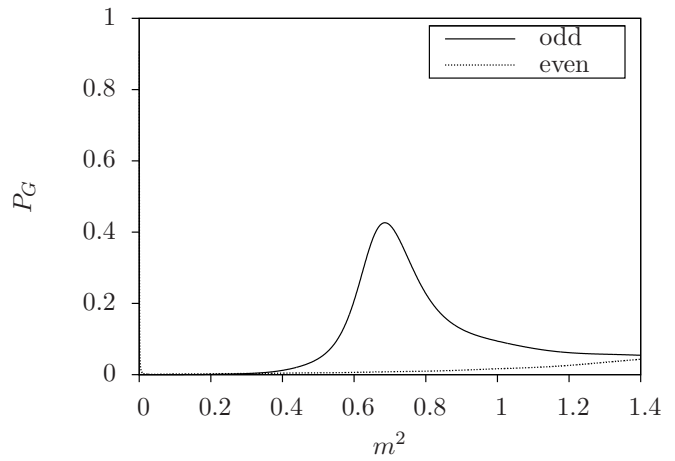

(b) $\xi=0.9, k=3$

FIG. 5: The profiles of $P_{\mathrm{G}}$ for massive even and odd gravity KK modes for the parameters $\xi=0.9$, $\phi_{0}=1, b=1, k=1$ and $k=3$.

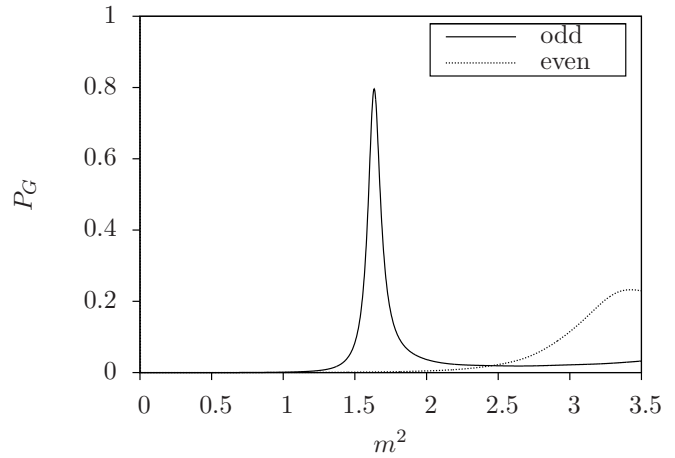

(a) $\xi=0.99, k=1$

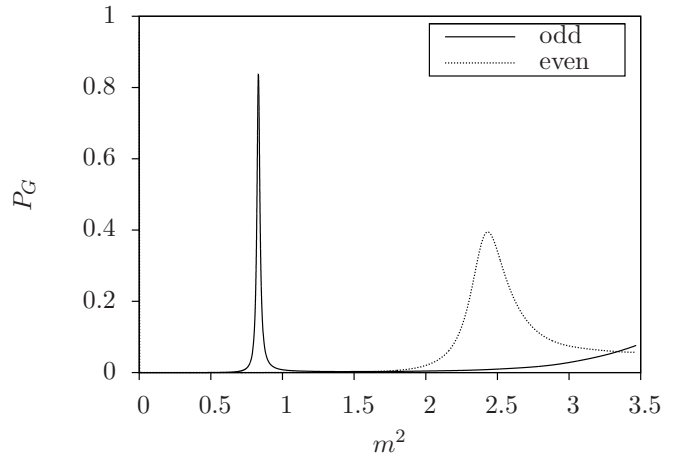

(b) $\xi=0.99, k=3$

FIG. 6: The profiles of $P_{\mathrm{G}}$ for massive even and odd gravity $\mathrm{KK}$ modes for the parameters $\xi=0.99, \phi_{0}=1, b=1, k=1$ and $k=3$.

\section{LOCALIZATION OF THE VARIOUS MATTERS ON THE THICK BRANES}

In this section, we will investigate the effect of nonminimally coupled parameter $\xi$ on the localization and resonances of various bulk matter fields on the thick branes, by presenting the potential of the corresponding Schrödinger equation for the KK modes of the various matter fields. Spin-0 scalars, spin-1 vectors and spin-1/2 fermions will be considered by means of the gravitational interaction. 


\begin{tabular}{|c|c|c|c|c|c|c|c|}
\hline$\xi$ & $k$ & Height of $V_{\mathrm{QM}}$ & $n$ & $m^{2}$ & $m$ & $\Gamma$ & $\tau$ \\
\hline \hline 0.9 & 3 & $V_{\mathrm{QM}}^{\max }=1.1322$ & 1 & 0.685649 & 0.82804 & 0.123867 & 8.07321 \\
\hline \hline 0.99 & 1 & $V_{\mathrm{QM}}^{\max }=2.8051$ & 1 & 1.634424 & 1.27845 & 0.041632 & 24.02015 \\
\cline { 2 - 7 } & 3 & $V_{\mathrm{QM}}^{\max }=2.7746$ & 1 & 0.831835 & 0.91205 & 0.013373 & 74.77949 \\
\cline { 4 - 7 } & & & 2 & 2.433646 & 1.56001 & 0.104536 & 9.56606 \\
\hline
\end{tabular}

TABLE I: The mass, width, and lifetime of the resonances of gravity. The parameters are set to $\phi_{0}=1$ and $b=1$. Here $n$ is the order of the resonant states with corresponding $m^{2}$ from small to large.

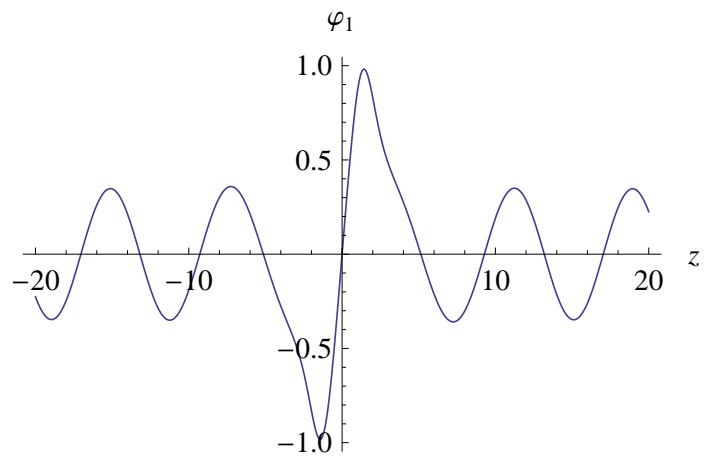

(a) $\xi=0.9, k=3, n=1$

FIG. 7: The wave function of the gravity resonant KK mode for the double-kink background scalar. The parameters are set to $\xi=0.9, \phi_{0}=1, b=1$ and $k=3$.

\section{A. Scalar fields and vector fields}

We first consider the localization of scalar and vector fields on the thick branes obtained in the previous section, then turn to fermions in the next subsections. Let us start by considering the action of a massless real scalar coupled to gravity

$$
S_{0}=-\frac{1}{2} \int d^{5} x \sqrt{-g} g^{M N} \partial_{M} \Phi \partial_{N} \Phi
$$

By using of the KK decomposition $\Phi(x, z)=\sum_{n} \phi_{n}(x) \chi_{n}(z) \mathrm{e}^{-3 A / 2}$, it is easy to derive the equations for the scalar KK modes:

$$
\left[-\partial_{z}^{2}+V_{0}(z)\right] \chi_{n}(z)=m_{n}^{2} \chi_{n}(z)
$$




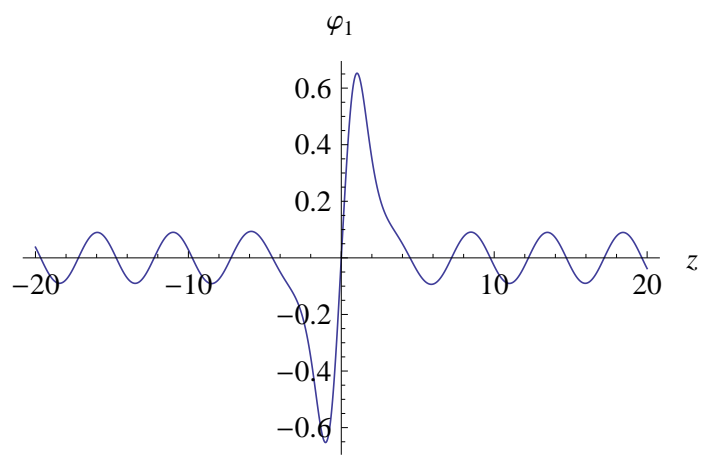

(a) $\xi=0.99, k=1, n=1$

FIG. 8: The wave function of the gravity resonant KK mode for the single-kink background scalar. The parameters are set to $\xi=0.99, \phi_{0}=1, b=1$ and $k=1$.

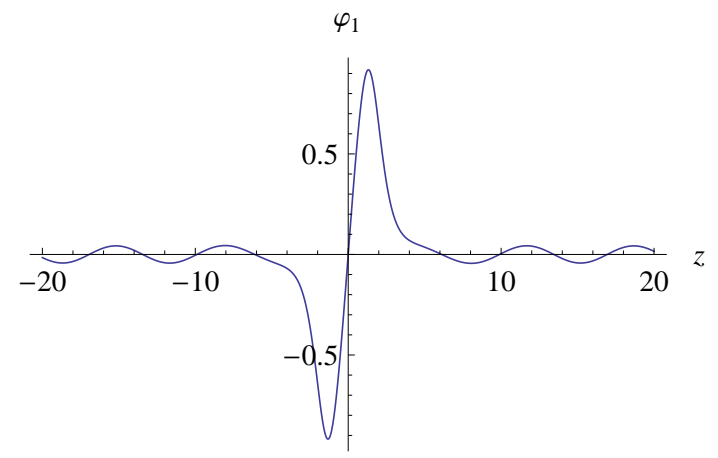

(a) $\xi=0.99, k=3, n=1$

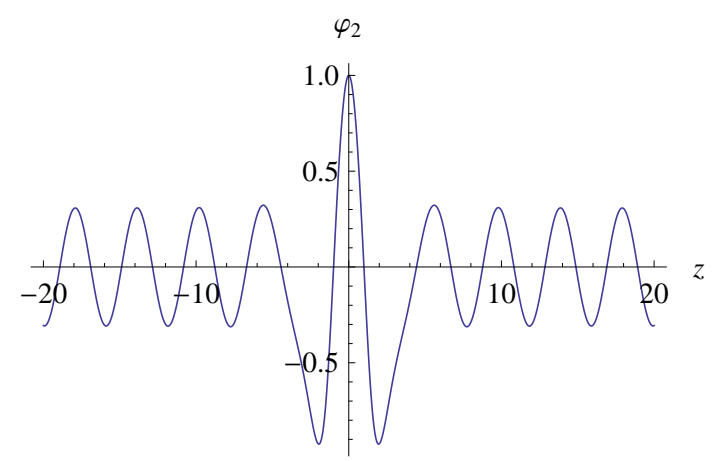

(b) $\xi=0.99, k=3, n=2$

FIG. 9: The wave functions of the gravity resonant KK modes for the double-kink background scalar. The parameters are $\xi=0.99, \phi_{0}=1, b=1$ and $k=3$.

where the Schrödinger potential is given by

$$
V_{0}(z)=\frac{3}{2} A^{\prime \prime}(z)+\frac{9}{4} A^{\prime 2}(z)
$$

Here $m_{n}$ are the masses of the scalar KK modes, they are also the masses of the fourdimensional scalars $\phi_{n}(x)$. It is clear that the potential $V_{0}(z)$ defined in (47) is a fourdimensional mass-independent potential. Note that those scalar KK modes localized on the branes should satisfies the following orthonormality conditions:

$$
\int_{-\infty}^{+\infty} \chi_{m}(z) \chi_{n}(z) d z=\delta_{m n}
$$


For the thick branes considered previous section, the warp factor can not be written as an analytical form, however, the potential $V_{0}$ can be solved numerically, which is shown in Figs. 10 and 11 .

The scalar zero mode can be solved from (46) by setting $m_{0}=0$ :

$$
\chi_{0} \propto \mathrm{e}^{\frac{3}{2} A(z)}
$$

This scalar zero mode (hence the four-dimensional massless scalar) is localized on the branes because the boundaries of the five-dimensional space-time along extra dimension are AdS. This can also be confirmed by the following simple calculation:

$$
\begin{gathered}
\chi_{0}(z \rightarrow \pm \infty) \rightarrow\left(\frac{1}{b|z|}\right)^{\frac{3}{2}}, \\
\int_{1}^{\infty}\left(\frac{1}{b|z|}\right)^{3} d z=\frac{1}{2 b^{3}}<\infty .
\end{gathered}
$$

In fact, this is a well known conclusion in brane-world model. Here, our point is another question: where the four-dimensional massless scalar locates when the thick branes split? As far as we know, when a thick brane splits into two sub-branes in minimally coupled theories, the four-dimensional massless scalar is located between the two sub-branes. Here, for the case of nonminimally coupled theory considered in this papaer, we can see from Figs. 10 and 11, as well as Eqs. (49), (15) and (16) that, the four-dimensional massless scalar is localized at the center of the two sub-branes generated by single-kink or between them by double-kink as $\xi<\xi_{0}$, just as the usual situation, while it is localized on each sub-brane when the coupling parameter $\xi$ is greater than its critical value $\xi_{0}$.

All other scalar KK modes are continuum and massive, for which we do not find resonant KK modes in the spectrum. This is different the case of gravity.

For Abelian spin-1 vectors described by the following action

$$
S_{1}=-\frac{1}{4} \int d^{5} x \sqrt{-g} g^{M N} g^{R S} F_{M R} F_{N S},
$$

because the asymptotical form of the warp factor is same as the RS brane-world when far away from the branes, with the same case of RS brane the vector zero mode can not localized on the branes with AdS boundaries. This problem can be solve in some higher dimensional models [49] or in thick dS branes and Weyl thick branes models [55]. We do not yet find vector resonant KK modes in the spectrum. 


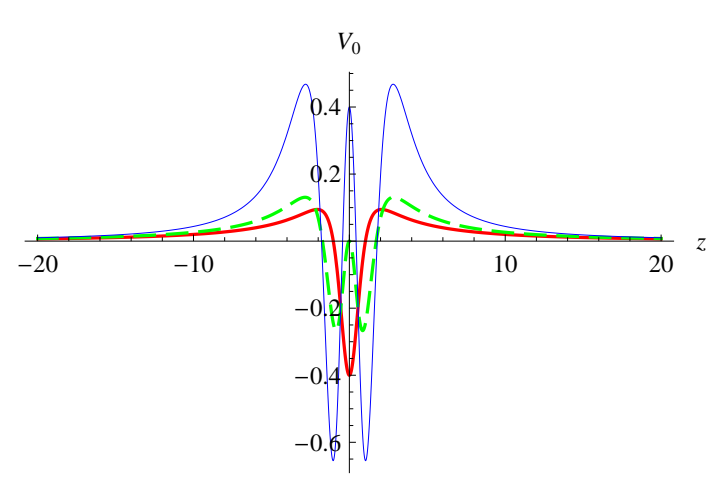

(a) $k=1$

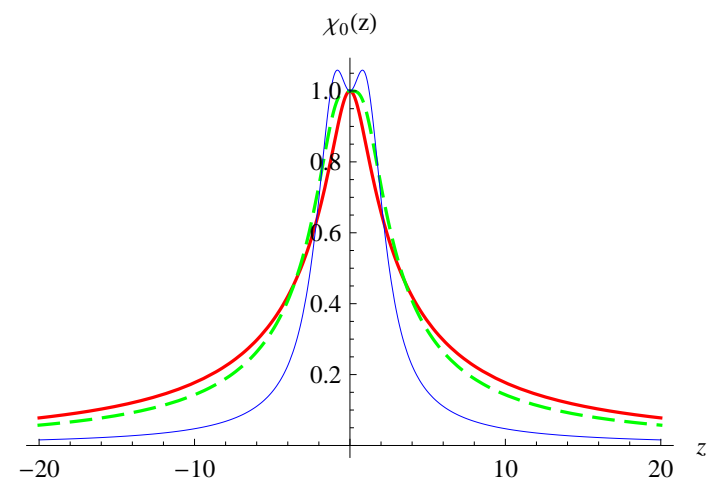

(b) $k=1$

FIG. 10: The shapes for the potential of the scalar KK modes $V_{0}(z)$ and the scalar zero mode $\chi_{0}(z)$. The parameters are set to $\xi=0.1$ for the thick red line, $\xi=0.5$ for the dashed green line, and $\xi=0.9$ for the thin blue line. The other parameters are set as $\phi_{0}=1, b=1$, and $k=1$.

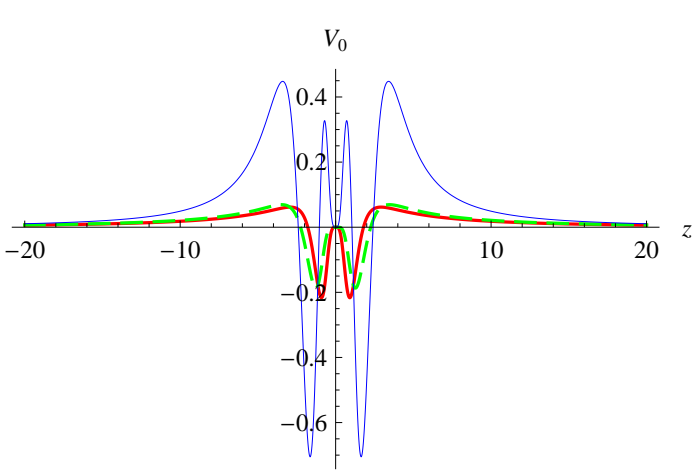

(a) $k=3$

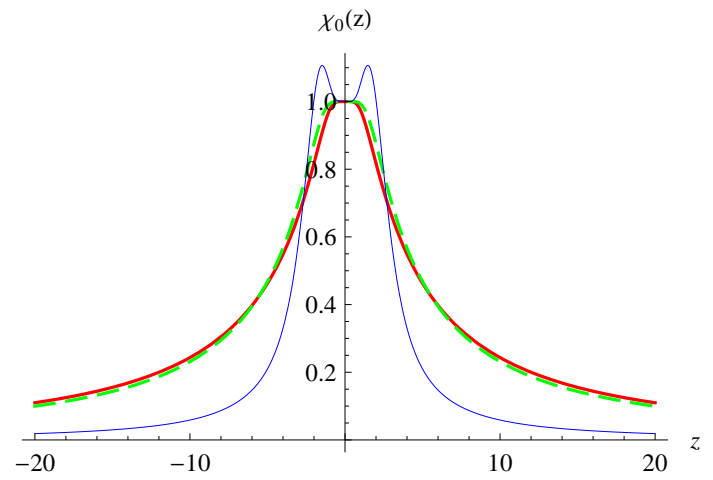

(b) $k=3$

FIG. 11: The shapes for the potential of the scalar KK modes $V_{0}(z)$ and the scalar zero mode $\chi_{0}(z)$. The parameters are set to $\xi=0.1$ for the thick red line, $\xi=0.3$ for the dashed green line, and $\xi=0.9$ for the thin blue line. The other parameters are set as $\phi_{0}=1, b=1$, and $k=3$.

\section{B. Spin-1/2 fermion fields}

Next, we will study the localization and resonances of fermions on the thick branes. In five-dimensional space-time, fermions are four-component spinors and their Dirac structure can be described by the curved space gamma matrices $\Gamma^{M}=e^{-A}\left(\gamma^{\mu}, \gamma^{5}\right)$, where $\gamma^{\mu}$ and $\gamma^{5}$ are the usual flat gamma matrices in the four-dimensional Dirac representation. The Dirac 
action of a massless spin-1/2 fermion coupled to the background scalar $\phi(12)$ is

$$
S_{1 / 2}=\int d^{5} x \sqrt{-g}\left(\bar{\Psi} \Gamma^{M} D_{\mu} \Psi-\eta \bar{\Psi} G(\phi) \Psi\right),
$$

where $\eta$ is a coupling constant, and $D_{\mu} \Psi=\left(\partial_{M}+\omega_{M}\right) \Psi$ with the nonvanishing components of the spin connection $\omega_{M}$ for the background metric (8) given by $\omega_{\mu}=\frac{1}{2} A^{\prime} \gamma_{\mu} \gamma_{5}$. Then the equation of motion reads

$$
\left[\gamma^{\mu} \partial_{\mu}+\gamma^{5}\left(\partial_{z}+2 A^{\prime}\right)-\eta \mathrm{e}^{A} G(\phi)\right] \Psi=0
$$

where $\gamma^{\mu} \partial_{\mu}$ is Dirac operator on the brane.

Now we would like to investigate the effect of the coupling parameter $\xi$ on the localization and resonances of the Dirac spinor on the branes by studying the above five-dimensional Dirac equation. Because of the Dirac structure of the fifth gamma matrix $\gamma^{5}$, we expect that the left- and right-chiral projections of the four-dimensional part have different behaviors. From the equation of motion (54), we will search for the solutions of the general chiral decomposition

$$
\Psi=\sum_{n}\left[\psi_{L, n}(x) L_{n}(z)+\psi_{R, n}(x) R_{n}(z)\right] \mathrm{e}^{-2 A},
$$

where $\psi_{L}=\frac{1-\gamma^{5}}{2} \psi$ and $\psi_{R}=\frac{1+\gamma^{5}}{2} \psi$ are the left- and right-chiral components of a fourdimensional Dirac field $\psi$, respectively. By demanding $\psi_{L, R}$ satisfy the four-dimensional massive Dirac equations $\gamma^{\mu}\left(\partial_{\mu}+\hat{\omega}_{\mu}\right) \psi_{L, R}=m \psi_{R, L}$, we obtain the following Schrödinger-like equations for the KK modes of the left- and right-chiral fermions:

$$
\begin{aligned}
& \left(-\partial_{z}^{2}+V_{L}(z)\right) L_{n}=m^{2} L_{n} \\
& \left(-\partial_{z}^{2}+V_{R}(z)\right) R_{n}=m^{2} R_{n}
\end{aligned}
$$

where the effective potentials are

$$
\begin{aligned}
V_{L}(z) & =\left(\eta \mathrm{e}^{A} G(\phi)\right)^{2}-\partial_{z}\left(\eta \mathrm{e}^{A} G(\phi)\right), \\
V_{R}(z) & =\left.V_{L}(z)\right|_{\eta \rightarrow-\eta} .
\end{aligned}
$$

For the purpose of getting the standard four-dimensional action for a massless fermion and an infinite sum of the massive fermions:

$$
\begin{aligned}
S_{\frac{1}{2}} & =\int d^{5} x \sqrt{-g} \bar{\Psi}\left[\Gamma^{M}\left(\partial_{M}+\omega_{M}\right)-\eta G(\phi)\right] \Psi \\
& =\sum_{n} \int d^{4} x \sqrt{-\hat{g}} \bar{\psi}_{n}\left[\gamma^{\mu}\left(\partial_{\mu}+\hat{\omega}_{\mu}\right)-m_{n}\right] \psi_{n}
\end{aligned}
$$


we need the following orthonormality conditions for $L_{n}$ and $R_{n}$ :

$$
\begin{aligned}
& \int_{-\infty}^{\infty} L_{m} L_{n} d z=\delta_{m n} \\
& \int_{-\infty}^{\infty} R_{m} R_{n} d z=\delta_{m n} \\
& \int_{-\infty}^{\infty} L_{m} R_{n} d z=0
\end{aligned}
$$

From Eqs. (56) and (57), it is clear that, in order to localize the left- and right-chiral fermions, some kind of scalar-fermion coupling must be introduced. This situation is similar to the one in Refs. [21, 48, 49, 52, 55, 60, 70], in which the authors introduced the scalar-fermion coupling term $m \bar{\Psi} F(\phi) \Psi$ for the localization of the fermion fields on a brane. Moreover, if we demand that $V_{L}(z)$ and $V_{R}(z)$ are $Z_{2}$-even with respect to the extra dimension coordinate $z, G(\phi)$ should be an odd function of the kink $\phi(z)$. In this paper, we choose the simplest Yukawa coupling: $G(\phi)=\phi$. So the potentials for left- and right-chiral fermion KK modes can be expression as

$$
\begin{aligned}
& V_{L}(z)=\eta^{2} \mathrm{e}^{2 A(z)} \phi^{2}(z)-\eta \mathrm{e}^{A} \phi^{\prime}(z)-\eta \phi(z) \mathrm{e}^{A(z)} A^{\prime}(z), \\
& V_{R}(z)=\eta^{2} \mathrm{e}^{2 A(z)} \phi^{2}(z)+\eta \mathrm{e}^{A} \phi^{\prime}(z)+\eta \phi(z) \mathrm{e}^{A(z)} A^{\prime}(z) .
\end{aligned}
$$

First, by considering the initial conditions of the warp factor and the background scalar at $z=0$, the values of the potentials can be obtained:

$$
\begin{aligned}
& V_{L}(0)=-\left.\eta \phi^{\prime}(z)\right|_{z=0}, \\
& V_{R}(0)=+\left.\eta \phi^{\prime}(z)\right|_{z=0},
\end{aligned}
$$

So for $k=1$,

$$
V_{L}(0)=-\eta \phi_{0} b, \quad V_{R}(0)=+\eta \phi_{0} b
$$

and for $k=3$,

$$
V_{L, R}(0)=0 .
$$

Second, when far away from the brane $z \rightarrow \pm \infty$,

$$
V_{L, R}(z \rightarrow \pm \infty) \rightarrow 0
$$


Here the potentials, which are shown in Figs. 12 and 13, can be solved by the numerical method.

The left- and right-chiral fermion zero modes are solved as

$$
\begin{aligned}
& L_{0}(z) \propto \exp \left(-\eta \int_{0}^{z} d z^{\prime} \mathrm{e}^{A\left(z^{\prime}\right)} \phi\left(z^{\prime}\right)\right), \\
& \left.R_{0}(z) \propto L_{0}(z)\right|_{\eta \rightarrow-\eta} .
\end{aligned}
$$

From the asymptotic behavior of the warp factor as $z \rightarrow \pm \infty$, the asymptotic behavior of left- and right-chiral fermion zero mode can be analyzed:

$$
\begin{aligned}
& L_{0}(z \rightarrow \pm \infty) \rightarrow|z|^{-\frac{\eta \phi_{0}}{b}}, \\
& R_{0}(z \rightarrow \pm \infty) \rightarrow|z|^{\frac{\eta \phi_{0}}{b}} .
\end{aligned}
$$

So for the positive coupling constant $\eta$, only the left-chiral zero mode tends to zero when far away from the branes, which may be localized on the brane. We need further check whether the normalization condition (59) is satisfied for the left-chiral zero mode, i.e.,

$$
\int_{-\infty}^{+\infty} d z L_{0}^{2}(z)<\infty
$$

Since the values of the zero modes are finite at finite $z$, the above normalization condition is equivalent to the following condition

$$
\int_{1}^{\infty} d z|z|^{-\frac{2 \eta \phi_{0}}{b}}<\infty
$$

Only when $\eta>\eta_{0}=\frac{b}{2 \phi_{0}}$ (note that $\phi_{0}$ and $b$ are positive real parameters), the above integral is convergent, which means that the left-chiral zero mode can be localized on the branes under this condition. From the shapes of the zero mode of left-chiral fermions (Fig. 14), it can be seen that the zero mode is localized on the center of the two sub-branes when the thick brane splits (single-kink case) or between them (double-kink case). However, we will show in the following that the nonminimally coupling parameter $\xi$ would effect the resonance spectrum of both the left- and right-chiral fermions.

All the massive KK modes of the left- and right-chiral fermions are the continuum modes, and can not be localized on the brane. Next, we are going to investigate the quasilocalization of the left- and right-chiral fermion KK modes, which are called resonances. As Sec. [II, the relative probability for finding the resonances on a brane can be given as

$$
P_{\mathrm{L}, \mathrm{R}}\left(m^{2}\right)=\frac{\int_{-z_{b}}^{z_{b}}|L, R(z)|^{2} d z}{\int_{-z_{\max }}^{z_{\max }}|L, R(z)|^{2} d z} .
$$




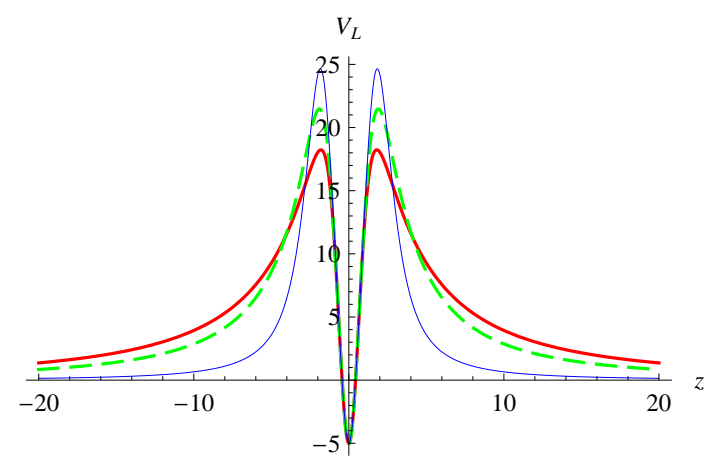

(a) $k=1$

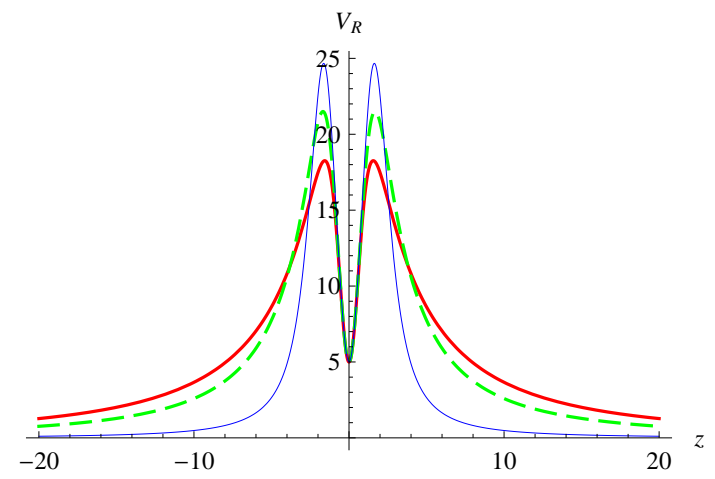

(b) $k=1$

FIG. 12: The shapes of the potentials for the left- and right-chiral fermions coupled with singlekink scalar $(k=1)$. The parameters are set to $\xi=0.1$ for the thick red line, $\xi=0.5$ for the dashed green line, and $\xi=0.9$ for the thin blue line. The other parameters are set to $\phi_{0}=1, \eta=5$, and $b=1$.

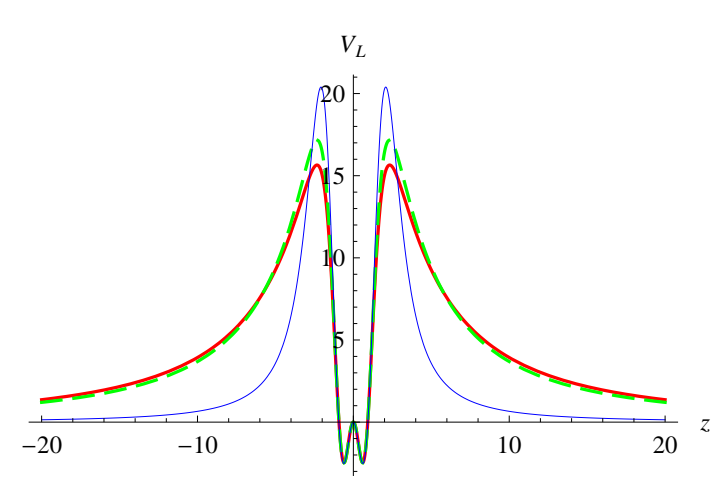

(a) $k=3$

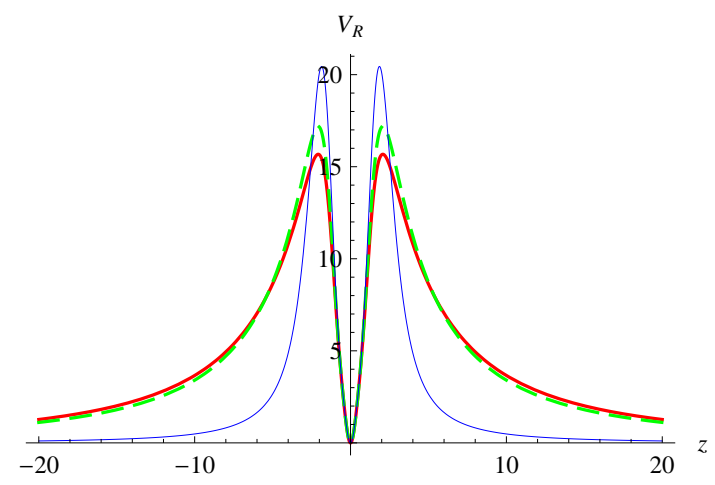

(b) $k=3$

FIG. 13: The shapes of the potentials for the left- and right-chiral fermions coupled with doublekink scalar $(k=3)$. The parameters are set to $\xi=0.1$ for the thick red line, $\xi=0.3$ for the dashed green line, and $\xi=0.9$ for the thin blue line. The other parameters are set to $\phi_{0}=1, \eta=5$, and $b=1$.

In order to investigate the effect of the gravity-scalar coupling constant $\xi$ on fermion resonances, we will investigate the resonant KK modes with different values of $\xi$.

For the single-kink background scalar field $(k=1)$, the mass, width and lifetime of the resonant KK modes with different values of $\xi$ are listed in Table II. As an example, we plot the shapes of $P_{\mathrm{L}, \mathrm{R}}\left(m^{2}\right)$ corresponding to $\xi=0.9$ and $k=1$ in Fig. 15. From Table 1 and 


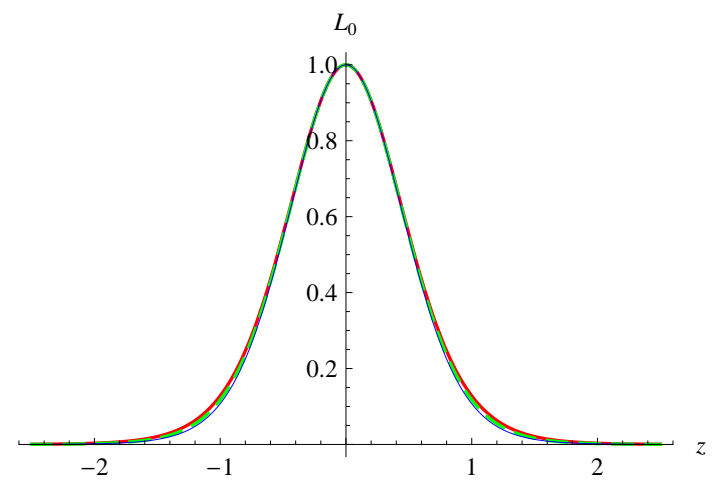

(a) $k=1$

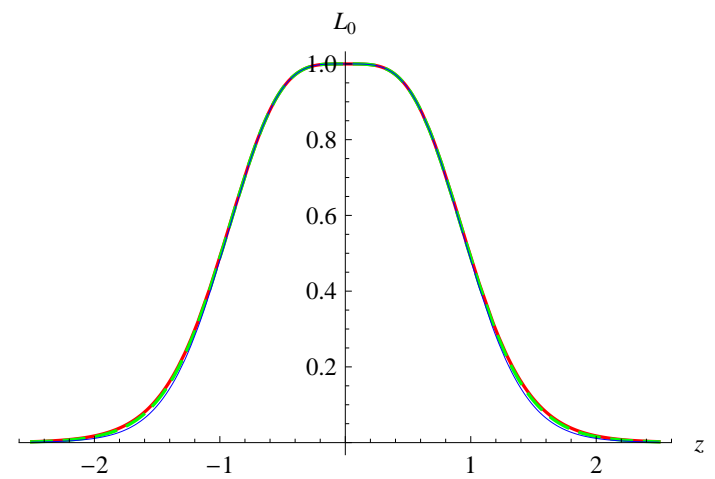

(b) $k=3$

FIG. 14: The shapes of the left-chiral fermion KK zero mode. The parameters are set to $\xi=0.1$ for the thick red line, $\xi=0.5$ for the dashed green line, and $\xi=0.9$ for the thin blue line for the left figure $(k=1)$, and the parameters are set to $\xi=0.1$ for the thick red line, $\xi=0.3$ for the dashed green line, and $\xi=0.9$ for the thin blue line for the right figure $(k=3)$. The other parameters are set to $\phi_{0}=1, \eta=5$, and $b=1$.

Fig. 15, it can be seen that the mass and lifetime of left- and right-chiral fermion resonances are almost the same, so the formation of the four-dimensional massive Dirac fermions can be realized [59]. The shapes of the resonances $L_{n}$ and $R_{n}$ are shown in Figs. 16 and 17 for $\xi=0.9$ and $k=1$. For the left-chiral KK resonances, it can be seen that the first resonant KK mode is an odd-parity wave function, and the second one has even parity. However, for the right-chiral KK resonances, the first resonant KK mode has even parity and the second one has odd parity. This is held for any $n$-th fermion resonances, namely, the parities of the $n$-th left- and right-chiral resonances are opposite. In fact, this conclusion is originated from relationship between the two potentials $V_{L}(z)$ and $V_{R}(z)$, or equivalently, from the coupled equations of the left- and right-chiral fermions, which are not given in this paper but one can refer [67]. Further, four-dimensional massive Dirac fermions can be obtained, which are consisted of the pairs of coupled left- and right-chiral KK modes with different parities.

From Table III, it is shown that the lifetimes of the first resonant KK modes satisfy $\tau_{n=1}(\xi=0.5)>\tau_{n=1}(\xi=0.1)>\tau_{n=1}(\xi=0.9)$. From Fig. 12, it is seen that the width of the barrier of the potentials in the vicinity of $V_{L, R}=m_{1}^{2}$ for $\xi=0.5$ is close to that for $\xi=0.1$ and larger than that for $\xi=0.9$, so $\tau_{n=1}(\xi=0.5)$ and $\tau_{n=1}(\xi=0.1)$ are larger than $\tau_{n=1}(\xi=0.9)$. Moreover, the widths of the barrier of the potentials for $\xi=0.5$ and $\xi=0.1$ 
are the same, but the height of the barrier for $\xi=0.5$ is larger than that for $\xi=0.1$, so $\tau_{n=1}(\xi=0.5)>\tau_{n=1}(\xi=0.1)$. For the second resonant KK modes, the situation is similar to the first ones. As Fig. 12 shown, the width of the barrier of the potentials in the vicinity of $V_{L, R}=m_{2}^{2}$ for $\xi=0.5$ is the largest, so $\tau_{n=2}(\xi=0.5)$ is the largest one. Hence, we can come to the conclusion that the lifetimes of the resonant KK modes are decided by the width and the hight of the barrier of the potential and the width is more important than the hight.

\begin{tabular}{|c|c|c|c|c|c|c|c|}
\hline$\xi$ & Chiral & Height of $V_{\mathrm{L}, \mathrm{R}}$ & $n$ & $m^{2}$ & $m$ & $\Gamma$ & $\tau$ \\
\hline \multirow[t]{4}{*}{0.1} & \multirow[t]{2}{*}{ Left } & \multirow[t]{2}{*}{$V_{L}^{\max }=14.036$} & 1 & 8.5206 & 2.9190 & 0.001222 & 818.64177 \\
\hline & & & 2 & 13.6638 & 3.6965 & 0.111288 & 8.98573 \\
\hline & \multirow[t]{2}{*}{ Right } & \multirow[t]{2}{*}{$V_{R}^{\max }=14.072$} & 1 & 8.5206 & 2.9190 & 0.001216 & 822.65478 \\
\hline & & & 2 & 13.6463 & 3.6941 & 0.111813 & 8.94354 \\
\hline \multirow[t]{4}{*}{0.5} & \multirow[t]{2}{*}{ Left } & \multirow[t]{2}{*}{$V_{L}^{\max }=16.611$} & 1 & 8.8961 & 2.9826 & 0.000725 & 1380.15215 \\
\hline & & & 2 & 15.1313 & 3.8899 & 0.063637 & 15.71416 \\
\hline & \multirow[t]{2}{*}{ Right } & \multirow[t]{2}{*}{$V_{R}^{\max }=16.651$} & 1 & 8.8961 & 2.9826 & 0.000722 & 1385.67372 \\
\hline & & & 2 & 15.1188 & 3.8883 & 0.064661 & 15.46516 \\
\hline \multirow[t]{4}{*}{0.9} & \multirow[t]{2}{*}{ Left } & \multirow[t]{2}{*}{$V_{L}^{\max }=18.411$} & 1 & 9.2353 & 3.0390 & 0.003074 & 325.2705 \\
\hline & & & 2 & 16.3330 & 4.0414 & 0.081694 & 12.2408 \\
\hline & \multirow[t]{2}{*}{ Right } & \multirow[t]{2}{*}{$V_{R}^{\max }=18.466$} & 1 & 9.2353 & 3.0390 & 0.003120 & 320.4962 \\
\hline & & & 2 & 16.3363 & 4.0418 & 0.084267 & 11.8671 \\
\hline
\end{tabular}

TABLE II: The mass, width, and lifetime of resonances for fermions. The parameters are set to $k=1, \eta=5, \phi_{0}=1$ and $b=1$. Here $n$ is the order of resonant states with corresponding $m^{2}$ from small to large.

For double-kink background scalar field $(k=3)$, because the mass, width and lifetime of the right-chiral fermion resonant KK modes is the same as that of left-chiral fermion resonances, we only list the mass, width and lifetime for the left-chiral fermion resonant KK modes with different values of $\xi$ are listed in Table III. It is known that the number of the resonant KK modes will increase when the coupling constant $\xi$ becomes larger. It is also 


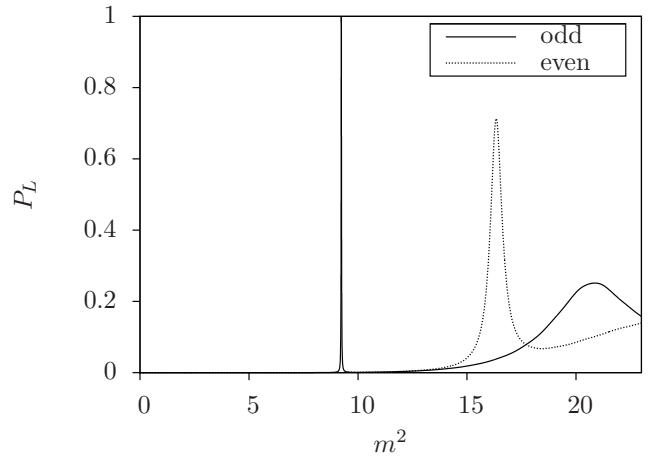

(a) $\xi=0.9, k=1$, Left-chiral

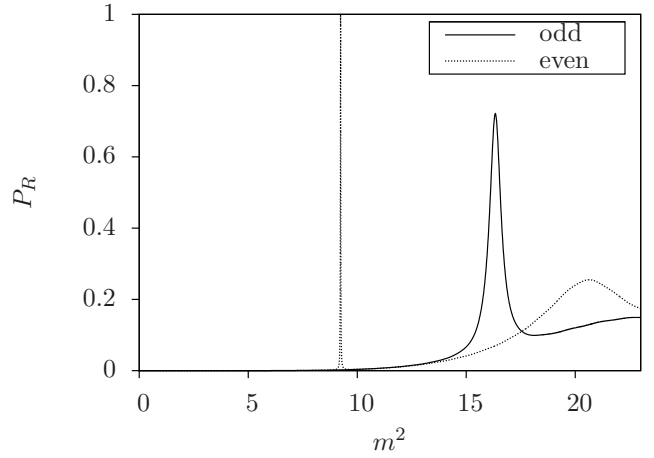

(b) $\xi=0.9, k=1$, Right-chiral

FIG. 15: The profiles of $P_{\mathrm{L}, \mathrm{R}}$ for the left- and right-chiral fermion resonances for the brane with the parameters $k=1, \xi=0.9, \eta=5, \phi_{0}=1$ and $b=1$.

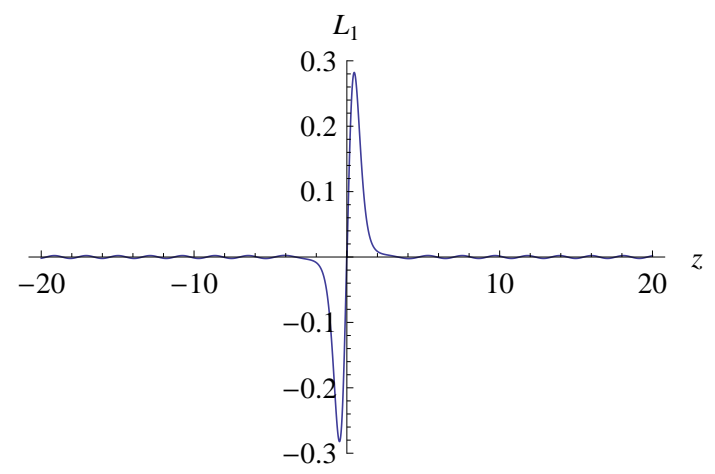

(a) $n=1$

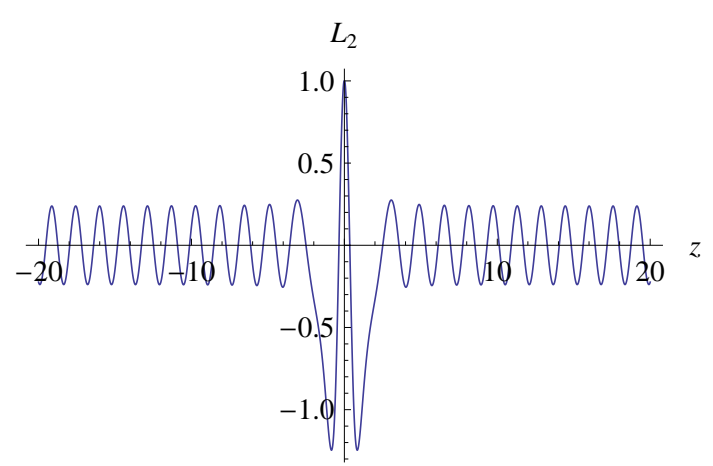

(b) $n=2$

FIG. 16: The profiles of the wave function for the left-chiral fermion resonances $L_{n}$ with the parameters $k=1, \xi=0.9, \eta=5, \phi_{0}=1$ and $b=1$.

shown that the lifetimes of the first resonant KK modes satisfy $\tau_{n=1}(\xi=0.1)>\tau_{n=1}(\xi=$ $0.3)>\tau_{n=1}(\xi=0.9)$, which is because that the widths of the barrier of the potentials in the vicinity of $V_{L, R}=m_{1}^{2}$ for $\xi=0.1$ are the largest and that for $\xi=0.9$ are the smallest from Fig. 13. For the other resonant KK modes, the situation is similar to the case of $k=1$.

\section{CONCLUSIONS AND DISCUSSIONS}

In this paper, we first review the model of thick branes with a nonminimally coupled background scalar field and then investigate the structure of the branes. In our model, 


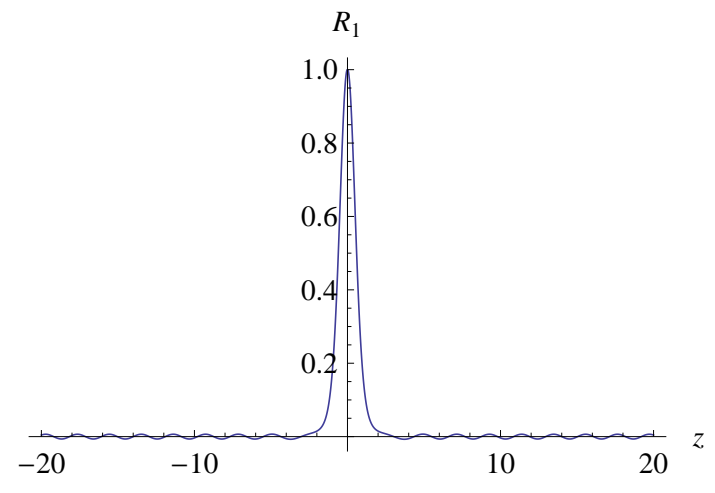

(a) $n=1$

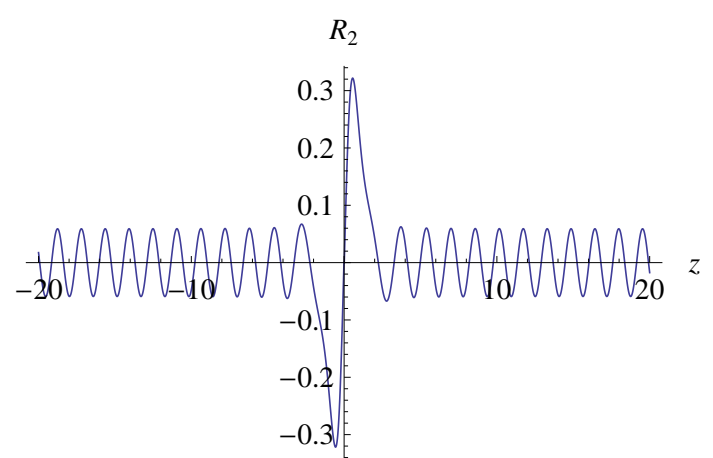

(b) $n=2$

FIG. 17: The profiles of the wave function for the right-chiral fermion resonances $R_{n}$ with the parameters $k=1, \xi=0.9, \eta=5, \phi_{0}=1$ and $b=1$.

the background scalar field is set as single-kink $(k=1)$ and double-kink $(k=3)$ solutions respectively. The nonminimally coupling between the gravity and the background scalar field is introduced via a term $\frac{1}{2} \xi R \phi^{2}$, and we find that the behaviors of the warp factor and the branes are related to the nonminimal coupling constant $\xi$. When the nonminimal coupling constant $\xi$ is smaller than its critical value $\xi_{0}$, the maximum of the warp factor is at $z=0$, and when $\xi>\xi_{0}$, the maxima of the warp factor are at both sides of $z=0$. For the single-kink $(k=1)$ case, the brane will split into two sub-branes with the increase of the nonminimal coupling constant $\xi$, and the distance of the two sub-branes increases with $\xi$. For the double-kink $(k=3)$ case, there are two sub-branes, and their distance also increases with $\xi$. The scalar potential $V(\phi)$ is a double well potential, and two vacua of the potential are at $\pm \phi_{0}$ for $\xi=0$ and not at $\pm \phi_{0}$ for $\xi>0$, respectively. Moreover, we investigate the effects of the nonminimal coupling constant on the localization of gravity and various bulk matter fields on the branes.

Firstly, the localization of gravity is considered. It is found that, for the case of singlekink $(k=1)$, the gravity zero mode is localized on the single brane with small $\xi$, and it is localized on the center of the two sub-branes with large $\xi$. For the case of double-kink $(k=3)$, the gravity zero mode is localized between the two sub-branes. All the massive modes are continuous spectrum wave functions and could not be localized on the branes. However, for larger coupling constant $\xi$, there could exist gravity resonant states, and the number of the resonances increases with $\xi$. 


\begin{tabular}{|c|c|c|c|c|c|c|c|}
\hline$\xi$ & Chiral & Height of $V_{\mathrm{L}}$ & $n$ & $m^{2}$ & $m$ & $\Gamma$ & $\tau$ \\
\hline \multirow[t]{3}{*}{0.1} & \multirow[t]{3}{*}{ Left } & \multirow[t]{3}{*}{$V_{L}^{\max }=15.648$} & 1 & 3.1973 & 1.7881 & $2.92 \times 10^{-10}$ & $3.42 \times 10^{9}$ \\
\hline & & & 2 & 8.8214 & 2.9701 & $1.45 \times 10^{-4}$ & $6.89 \times 10^{3}$ \\
\hline & & & 3 & 13.3115 & 3.6485 & 0.018426 & 54.2705 \\
\hline \multirow[t]{3}{*}{0.3} & \multirow[t]{3}{*}{ Left } & \multirow[t]{3}{*}{$V_{L}^{\max }=17.160$} & 1 & 3.2171 & 1.79363 & $3.91 \times 10^{-10}$ & $2.56 \times 10^{9}$ \\
\hline & & & 2 & 8.9598 & 2.9933 & $8.18 \times 10^{-5}$ & $1.22 \times 10^{4}$ \\
\hline & & & 3 & 13.7770 & 3.7117 & 0.010634 & 94.04994 \\
\hline \multirow[t]{4}{*}{0.9} & \multirow[t]{4}{*}{ Left } & \multirow[t]{4}{*}{$V_{L}^{\max }=20.395$} & 1 & 3.2735 & 1.8093 & $9.03 \times 10^{-6}$ & $1.11 \times 10^{5}$ \\
\hline & & & 2 & 9.3293 & 3.05439 & 0.00114 & 878.85943 \\
\hline & & & 3 & 14.9016 & 3.8603 & 0.01752 & 57.07182 \\
\hline & & & 4 & 19.8003 & 4.44975 & 0.128982 & 7.75318 \\
\hline
\end{tabular}

TABLE III: The mass, width, and lifetime of resonances for left-chiral fermion. The parameters are set to $k=3, \eta=5, \phi_{0}=1$ and $b=1$. Here $n$ is the order of resonant states with corresponding $m^{2}$ from small to large.

For scalar field, the zero mode is localized on the center of the branes ( $k=1$ case) or between them ( $k=3$ case) when $\xi<\xi_{0}$, while it is localized on each sub-brane when $\xi>\xi_{0}$. For the vector field, the zero mode can not be localized on the branes. All the massive modes for scalars and vectors are continuous spectrum wave functions and can not localized on the branes. There is not exist the resonant state for both fields.

For spin-1/2 fermion fields, in order to localized the left- and right-chiral fermions, we introduce the usual Yukawa coupling $\eta \bar{\Psi} \phi \Psi$. We find that for positive Yukawa coupling constant $\eta$ larger than its critical value $\eta_{0}$, the left-chiral fermion zero mode can be localized on the branes. For the case of $k=1$, the left-chiral fermion zero is localized on the single brane with small $\xi$ and on the center of the two sub-branes with large $\xi$. For the case of $k=3$, the left-chiral fermion zero is localized between the two sub-branes. The massive KK modes asymptotically turn into continuous plane waves when far away from the branes. It is interesting that the well of the potentials for left- and right-chiral fermions becomes deeper and deeper with the increase of the nonminimal coupling constant $\xi$, which leads to a series 
of massive fermions with a finite lifetime on the branes. The spectra of left- and right-chiral fermion resonances are the same, which demonstrates that a Dirac fermion with a finite lifetime on the branes can be composed from the left- and right-chiral fermion resonant KK modes.

At the end of this paper, we discuss the effect of the nonminimal coupling constant $\xi$ on the localization of gravity and various matter fields. For these zero modes, the situation is similar to the case of minimal coupling, i.e., only the vector zero mode is not localized on the branes, and all the other zero modes are localized on the branes. This indicates that the nonminimal coupling constant $\xi$ does not effect the localization of these zero modes. It is because that the localization of the zero modes is decided by the behavior of the system at the infinity of the extra dimension. The behavior of the system for the case of the nonminimal coupling, is similar to the one for the case of minimal coupling when far away from the branes. This can also be seen from the action (11). When the extra dimensional coordinate tends to infinity, $\phi( \pm \infty) \rightarrow \pm \phi_{0}$, and the action (1) is reduced to the following form

$$
S=\int d^{5} x \sqrt{-g}\left[\frac{\zeta}{2 \kappa_{5}^{2}} R-\frac{1}{2} g^{M N} \partial_{M} \phi \partial_{N} \phi-V(\phi)\right]
$$

with the constant $\zeta=\left(1-\kappa_{5}^{2} \xi \phi_{0}^{2}\right)$, which is similar to the general action of the thick brane for the case of minimal coupling. Therefore, the behavior of the zero modes is similar to these for the case of minimal coupling at $z \rightarrow \pm \infty$. However, near the branes, the effects of the nonminimal coupling constant $\xi$ are very obvious for the localization of gravity and matter fields. For the scalar zero mode, when $\xi$ is large, the zero mode is localized on each sub-brane. For the gravity and the fermion field, the number of the resonances increases with $\xi$.

\section{Acknowledgement}

The authors are extremely grateful for the anonymous referee, whose comments led to the improvement of this paper. This work was supported by the Program for New Century Excellent Talents in University, the Huo Ying-Dong Education Foundation of Chinese Ministry of Education (Grant No. 121106), the National Natural Science Foundation of China (Grant No. 11075065), the Doctoral Program Foundation of Institutions of Higher 
Education of China (Grant No. 20090211110028), and the Fundamental Research Funds for the Central Universities (Grant No. lzujbky-2012-k30).

[1] V.A. Rubakov and M.E. Shaposhnikov, Phys. Lett. B 125 (1983) 136; V.A. Rubakov and M.E. Shaposhnikov, Phys. Lett. B 125 (1983) 139; E.J. Squires, Phys. Lett. B 167 (1986) 286.

[2] M. Visser, Phys. Lett. B 159 (1985) 22.

[3] N. Arkani-Hamed, S. Dimopoulos and G. Dvali, Phys. Lett. B 429 (1998) 263; I. Antoniadis, N. Arkani-Hamed, S. Dimopoulos and G. Dvali, Phys. Lett. B 436 (1998) 257; N. ArkaniHamed, S. Dimopoulos, G. Dvali and N. Kaloper, Phys. Rev. Lett. 84 (2000) 586.

[4] S. Randjbar-Daemi and C. Wetterich, Phys. Lett. B 166 (1986) 65.

[5] L. Randall and R. Sundrum, Phys. Rev. Lett. 83 (1999) 3370; Phys. Rev. Lett. 83 (1999) 4690 .

[6] J. Lykken and L. Randall, JHEP 0006 (2000) 014.

[7] I. Antoniadis, Phys. Lett. B 246 (1990) 377.

[8] N. Arkani-Hamed, S. Dimopoulos, N. Kaloper and R. Sundtrum, Phys. Lett. B 480 (2000) 193; S. Kachru, M. Schulz and E. Silverstein, Phys. Rev. D 62 (2000) 045021; A. Kehagias, Phys. Lett. B 600 (2004) 133.

[9] O. DeWolfe, D.Z. Freedman, S.S. Gubser and A. Karch, Phys. Rev. D 62 (2000) 046008.

[10] D. Stojkovic, Phys. Rev. D 63 (2000) 025010.

[11] M. Gremm, Phys. Lett. B 478 (2000) 434; Phys. Rev. D 62 (2000) 044017; K. Ghoroku and M. Yahiro, hep-th/0305150; A. Kehagias and K. Tamvakis, Mod. Phys. Lett. A 17 (2002) 1767; Phys. Lett. B 504(2001) 38; M. Giovannini, Phys. Rev. D 64 (2001) 064023; Phys. Rev. D 65 (2002) 064008.

[12] S. Kobayashi, K. Koyama and J. Soda, Phys. Rev. D 65 (2002) 064014.

[13] C. Csaki, J. Erlich, T. Hollowood and Y. Shirman, Nucl. Phys. B 581 (2000) 309.

[14] G. Dvali, G. Gabadadze and M. Porrati, Phys. Lett. B 485 (2000) 208.

[15] R. Gregory, V.A. Rubakov and S.M. Sibiryakov, Phys. Rev. Lett. 84, (2000) 5928.

[16] A. Campos, Phys. Rev. Lett. 88 (2002) 141602.

[17] A. Wang, Phys. Rev. D66(2002) 024024.

[18] A. de Souza Dutra, A.C. Amaro de Faria, Jr, and M. Hott, Phys. Rev. D 78 (2008) 043526; D. Bazeia and A.R. Gomes, JHEP 0405 (2004) 012; A. Alonso Izquierdo, M. A. González León and J. Mateos Guilarte, Phys. Rev. D 65 (2002) 085012.

[19] R. Emparan, R. Gregory and C. Santos, Phys. Rev. D 63 (2001) 104022; R. Guerrero, A. Melfo 
and N. Pantoja, Phys. Rev. D 65 (2002) 125010; A. Melfo, N. Pantoja and A. Skirzewski, Phys. Rev. D 67 (2003) 105003; K.A. Bronnikov and B.E. Meierovich, Grav. Cosmol. 9 (2003) 313; O. Castillo-Felisola, A. Melfo, N. Pantoja and A. Ramirez, Phys. Rev. D 70 (2004) 104029.

[20] V. Dzhunushaliev, V. Folomeev, D. Singleton and S. Aguilar-Rudametkin, Phys. Rev. D 77 (2008) 044006; V. Dzhunushaliev, V. Folomeev, K. Myrzakulov and R. Myrzakulov, Gen. Rel. Grav. 41 (2009) 131; D. Bazeia, F.A. Brito and J.R. Nascimento, Phys. Rev. D 68 (2003) 085007; D. Bazeia, F.A. Brito and A.R. Gomes, JHEP 0411 (2004) 070; D. Bazeia, F.A. Brito and L. Losano, JHEP 0611 (2006) 064; D. Bazeia, A.R. Gomes and L. Losano, Int. J. Mod. Phys. A 24 (2009) 1135.

[21] T.R. Slatyer and R.R. Volkas, JHEP 0704 (2007) 062; R. Davies, D.P. George and R.R. Volkas, Phys. Rev. D 77 (2008) 124038.

[22] T. Gherghetta and M. Shaposhnikov, Phys. Rev. Lett. 85 (2000) 240.

[23] I.P. Neupane, JHEP 0009 (2000) 040; I.P. Neupane, Class. Quant. Grav. 19 (2002) 5507.

[24] O. Arias, R. Cardenas and I. Quiros, Nucl. Phys. B 643 (2002) 187; N. Barbosa-Cendejas and A. Herrera-Aguilar, JHEP 0510 (2005) 101; N. Barbosa-Cendejas and A. Herrera-Aguilar, Phys. Rev. D 73 (2006) 084022.

[25] M. Cvetic, S. Griffies and S.-J. Rey, Nucl. Phys. B381 (1992) 301; M. Cvetic and H.H. Soleng, Phys. Rept. 282 (1997) 159; M. Cvetic and M. Robnik, Phys. Rev. D77 (2008) 124003.

[26] N. Barbosa-Cendejas, A. Herrera-Aguilar, M.A. Reyes Santos and C. Schubert, Phys. Rev. D 77 (2008) 126013.

[27] N. Barbosa-Cendejas, A. Herrera-Aguilar, U. Nucamendi and I. Quiros, arXiv:0712.3098[hepth].

[28] S. Kobayashi, K. Koyama and J. Soda, Phys. Rev. D 65 (2002) 064014.

[29] A. Karch and L. Randall, JHEP 0105 (2001) 008.

[30] A. Herrera-Aguilar, D. Malagon-Morejon, R.R. Mora-Luna and U. Nucamendi, Mod. Phys. Lett. A25 (2010) 2089.

[31] V.I. Afonso, D. Bazeia and L. Losano, Phys. Lett. B634 (2006) 526.

[32] Y.-X. Liu, K. Yang and Y. Zhong JHEP 1010 (2010) 069.

[33] A. Brandhuber and K. Sfetsos, JHEP 9910 (1999) 013.

[34] J.I. Diaz, J. Negro, L.M. Nieto, O. Rosas-Ortiz, J. Phys. A: Math. Gen. 32 (1999) 8447.

[35] I. Oda, Phys. Rev. D64 (2001) 026002.

[36] Y.-X. Liu, Y. Zhong and K. Yang, Europhys. Lett. 90 (2010) 51001.

[37] A. Balcerzak and M.P. Dạbrowski, Phys. Rev. D81 (2010) 123527.

[38] Y. Zhong, Y.-X. Liu and K. Yang, Phys. Lett. B699 (2011) 398. 
[39] M. Gogberashvili and D. Singleton, Phys. Rev. D69 (2004) 026004; M. Gogberashvili and D. Singleton, Phys. Lett. B582 (2004) 95.

[40] H. Guo, Y.-X. Liu, S.-W. Wei and C.-E. Fu, Europhys. Letters 97 (2012) 60003.

[41] A. Herrera-Aguilar, D. Malagon-Morejon and R.R. Mora-Luna, JHEP 1011 (2010) 015; H. Guo, A. Herrera-Aguilar, Y.-X. Liu, D. Malagon-Morejon and R.R. Mora-Luna, arXiv:1103.2430 [hep-th].

[42] S. SenGupta, arXiv:0812.1092[hep-th].

[43] V. Dzhunushaliev, V. Folomeev, and M. Minamitsuji, Rept. Prog. Phys. 73 (2010) 066901.

[44] M. Shifman, Int. J. Mod. Phys. A25 (2010) 199.

[45] T.G. Rizzo, AIP Conf. Proc. 1256 (2010) 27.

[46] P.D. Mannheim, Brane-localized gravity, World Scientific Publishing Company, Singapore (2005).

[47] R. Maartens and K. Koyama, Living Rev. Rel. 13 (2010) 5.

[48] B. Bajc and G. Gabadadze, Phys. Lett. B 474 (2000) 282.

[49] I. Oda, Phys. Lett. B 496 (2000) 113.

[50] Y. Grossman and N. Neubert, Phys. Lett. B 474 (2000) 361; R. Koley and S. Kar, Phys. Lett. B 623 (2005) 244; A. Melfo, N. Pantoja and J.D. Tempo, Phys. Rev. D 73 (2006) 044033.

[51] S. Ichinose, Phys. Rev. D 66 (2002) 104015.

[52] C. Ringeval, P. Peter and J.P. Uzan, Phys. Rev. D 65 (2002) 044016.

[53] S. Randjbar-Daemi and M. Shaposhnikov, Phys. Lett. B 492 (2000) 361.

[54] R. Koley and S. Kar, Class. Quant. Grav. 22 (2005) 753.

[55] Y.-X. Liu, X.-H. Zhang, L.-D. Zhang and Y.-S. Duan, JHEP 0802 (2008) 067; Y.-X. Liu, L.-D. Zhang, L.-J. Zhang and Y.-S. Duan, Phys. Rev. D 78, 065025 (2008).

[56] S.L. Dubovsky, V.A. Rubakov and P.G. Tinyakov, Phys. Rev. D 62 (2000) 105011.

[57] Y. Brihaye and T. Delsate, Phys. Rev. D 78 (2008) 025014.

[58] Y.-X. Liu, L. Zhao and Y.-S. Duan, JHEP 0704 (2007) 097; Y.-X. Liu, L. Zhao, X.-H. Zhang and Y.-S. Duan, Nucl. Phys. B 785 (2007) 234; Y.-Q. Wang, T.-Y. Si, Y.-X. Liu and Y.-S. Duan, Mod. Phys. Lett. A 20 (2005) 3045; L. Zhao, Y.-X. Liu and Y.-S. Duan, Mod. Phys. Lett. A 23 (2008) 1129.

[59] C.A.S. Almeida, R. Casana, M.M. Ferreira and A.R. Gomes, Phys. Rev. D 79 (2009) 125022.

[60] Y.-X. Liu, J. Yang, Z.-H. Zhao, C.-E. Fu and Y.-S. Duan, Phys. Rev. D 80 (2009) 065019; Y.-X. Liu, C.-E. Fu, L. Zhao and Y.-S. Duan, Phys. Rev. D 80 (2009) 065020.

[61] R. Koley, J. Mitra and S. SenGupta, Phys. Rev. D 79 (2009) 041902(R).

[62] Y. Kodama, K. Kokubu and N. Sawado, Phys. Rev. D 79, 065024 (2009).

[63] Y.-X. Liu, Z.-H. Zhao, S.-W. Wei and Y.-S. Duan, JCAP 02 (2009) 003. 
[64] Y.-X. Liu, L.-D. Zhang, S.-W. Wei and Y.-S. Duan, JHEP 0808 (2008) 041.

[65] S. Aguilar and D. Singleton, Phys. Rev. D73 (2006) 085007; M. Gogberashvili, P. Midodashvili and D. Singleton, JHEP 0708 (2007) 033.

[66] R. Jackiw and C. Rebbi, Phys. Rev. D 13 (1976) 3398.

[67] Y.-X. Liu, H.-T. Li, Z.-H. Zhao, J.-X. Li and J.-R. Ren, JHEP 0910 (2009) 091.

[68] Y.-X. Liu, H. Guo, C.-E Fu and J.-R. Ren, JHEP 1002 (2010) 080.

[69] Y.-X. Liu, C.-E. Fu, H. Guo, S.-W. Wei and Z.-H. Zhao, JCAP 1012 (2010) 031; H.-T. Li, Y.-X. Liu, Z.-H. Zhao and H. Guo, Phys. Rev. D83 (2011) 045006.

[70] Z.-H. Zhao, Y.-X. Liu and H.-T. Li, Class. Quantum Grav. 27 (2010) 185001.

[71] C.-E. Fu, Y.-X. Liu and H. Guo, Phys. Rev. D84 (2011) 044036.

[72] Z.-H. Zhao, Y.-X. Liu, H.-T. Li and Y.-Q. Wang, Phys. Rev. D82 (2010) 084030; Z.-H. Zhao, Y.-X. Liu, Y.-Q. Wang and H.-T. Li, JHEP 1106 (2011) 045.

[73] Y.-X. Liu, C.-E. Fu, H. Guo, and H.-T. Li, Phys. Rev. D85 (2012) 084023.

[74] A. E. R. Chumbes, A. E. O. Vasquez, and M. B. Hott, Phys. Rev. D 83 (2011) 105010.

[75] K. Farakos and P. Pasipoularides, Phys. Rev. D 73 (2006) 084012.

[76] K. Farakos and P. Pasipoularides, Phys. Lett. B 621 (2005) 224.

[77] C. Bogdanos, A. Dimitriadis, and K. Tamvakis , Phys. Rev. D 74 (2006) 045003.

[78] A.A. Andrianov and L. Vecchi, Phys. Rev. D77 (2008) 044035.

[79] K. Farakos, G. Koutsoumbas, and P. Pasipoularides, Phys. Rev. D 76 (2007) 064025.

[80] A. Herrera-Aguilar, D. Malagon-Morejon, R.R. Mora-Luna and I. Quiros, Class. Quantum Grav. 29 (2012) 035012.

[81] M.R. Setare and E.N. Saridakis JCAP 0903 (2009) 002.

[82] N. Arkani-Hamed and M. Schmaltz, Phys. Rev. D 61 (2000) 033005; N. Arkani-Hamed, Y. Grossman and M. Schmaltz, Phys. Rev. D 61 (2000) 115004.

[83] D.-C. Dai, G. D. Starkman and D. Stojkovic, Phys. Rev. D 73 (2006) 104037. 